\title{
Hodge and signature theorems for a family of manifolds with fibre bundle boundary
}

\author{
EUGÉNIE HUNSICKER
}

\begin{abstract}
Over the past fifty years, Hodge and signature theorems have been proved for various classes of noncompact and incomplete Riemannian manifolds. Two of these classes are manifolds with incomplete cylindrical ends and manifolds with cone bundle ends, that is, whose ends have the structure of a fibre bundle over a compact oriented manifold, where the fibres are cones on a second fixed compact oriented manifold. In this paper, we prove Hodge and signature theorems for a family of metrics on a manifold $M$ with fibre bundle boundary that interpolates between the incomplete cylindrical metric and the cone bundle metric on $M$. We show that the Hodge and signature theorems for this family of metrics interpolate naturally between the known Hodge and signature theorems for the extremal metrics. The Hodge theorem involves intersection cohomology groups of varying perversities on the conical pseudomanifold $X$ that completes the cone bundle metric on $M$. The signature theorem involves the summands $\tau_{i}$ of Dai's $\tau$ invariant [10] that are defined as signatures on the pages of the Leray-Serre spectral sequence of the boundary fibre bundle of $M$. The two theorems together allow us to interpret the $\tau_{i}$ in terms of perverse signatures, which are signatures defined on the intersection cohomology groups of varying perversities on $X$.
\end{abstract}

14F40, 55N33, 14F43; 58J10, 13D22, 32S20

\section{Introduction}

The Hodge theorem and the Hirzebruch signature theorem form an important bridge between geometric and topological properties of compact smooth manifolds. As early as the 1950's mathematicians started to consider analogues of these theorems in more general settings. Building on work of Gaffney, Conner proved a Hodge theorem for manifolds with boundary in 1956 [9]. This theorem identified the spaces of $L^{2}$ harmonic forms under two different conditions at the boundary of the manifold with the absolute and relative de Rham cohomologies of the manifold. In the early 70's, Atiyah, Patodi and Singer [1] proved a signature theorem for manifolds with cylindrical metric near a boundary, and related it to Hodge and signature theorems for noncompact manifolds with cylindrical ends, that is, manifolds that off a compact set are isometric 
to $(0, \infty) \times N$ for some compact manifold $N$. Denote the original compact manifold with boundary by $\bar{M}$ and denote by $\widehat{M}$, the manifold obtained by smoothly gluing the half cylinder on $N=\partial \bar{M}$ to $\bar{M}$. The Atiyah-Patodi-Singer noncompact Hodge result says that the space of $L^{2}$ harmonic forms on $\widehat{M}$ is canonically isomorphic to the image of the relative cohomology of $\hat{M}$ in its absolute cohomology, ie

$$
\mathcal{H}_{(2)}^{*}(\widehat{M}, g) \cong \operatorname{Im}\left(H_{0}^{*}(\widehat{M}) \longrightarrow H^{*}(\widehat{M})\right) \cong \operatorname{Im}\left(H^{*}(\bar{M}, N) \longrightarrow H^{*}(\bar{M})\right) .
$$

Their signature result says first that the topological signature of $\bar{M}$ is equal to the signature of the pairing on middle degree $L^{2}$ harmonic forms on $\hat{M}$ given by integration, and secondly that both satisfy

$$
\sigma(M)=\int_{M} L(p(M, N))-\eta(N)(0)
$$

where $L$ is the Hirzebruch $L$-polynomial in the relative Pontrjagin classes on $M$ and $\eta$ is a spectral invariant of $N$.

The connection between $L^{2}$ Hodge theorems and intersection cohomology was made first by Cheeger in [5] at the same time as the dual intersection homology theory was being defined by Goresky and MacPherson [11]. A year later, after defining $L^{2}$ cohomology and studying its relationship to $L^{2}$ harmonic forms, Cheeger [6] showed that for a pseudomanifold $X$ with conical singularities and only even codimensional strata, the space of $L^{2}$ harmonic forms on the regular set is isomorphic to the middle perversity intersection cohomology, the unique intersection cohomology that satisfies Poincare duality, that is,

$$
H_{2}^{i}\left(X^{\mathrm{reg}}, g_{\text {cone }}\right) \cong I H_{\mathfrak{m}}^{i}(X)
$$

where $g_{\text {cone }}$ denotes a metric that near the singularities is a recursively defined conical metric.

In [5], Cheeger also recognized the important fact that in $L^{2}$ Hodge theorems for singular manifolds with even dimensional strata, it is necessary to impose "boundary" conditions at the singularity, just as we must consider boundary conditions for standard manifolds with boundary. Whereas in the complete case, there is a unique closed extension of the exterior derivative $d$, in the incomplete or singular, case, there may be several different closed extensions corresponding to different so-called "ideal boundary conditions." These correspond to several different $L^{2}$ cohomologies and several different closed self-adjoint extensions of the Laplace operator. For instance, in the case of a compact manifold with boundary, $\bar{M}$, the exterior derivative may have a number of closed extensions interpolating between the so-called maximal and minimal extensions. The cohomology corresponding to the maximal extension of $d$ is the 
absolute cohomology on $\bar{M}$. The first part of the theorem in Conner [9] shows that its classes are naturally represented by $L^{2}$ harmonic forms satisfying a version of Neumann boundary conditions. The complex given by the minimal extension of $d$ generates cohomology on $\bar{M}$ relative to its boundary. The second part of Conner's theorem shows that its classes are naturally represented by harmonic $L^{2}$ forms satisfying Dirichlet boundary conditions. The even codimension condition in the Hodge theorem for conical pseudomanifolds in [6] avoids this complication. It also guarantees that the space has a unique (Poincaré dual) middle perversity intersection cohomology. In [5], Cheeger discussed cases where even pseudomanifolds with even dimensional strata could satisfy Poincaré duality if appropriate ideal boundary conditions are chosen.

In the 1990's, new work was done on the eta invariant defined by Atiyah, Patodi and Singer. A number of mathematicians began to study its behavior in a family of fibre bundle metrics that become singular. Bismut and Cheeger [3], Melrose and Mazzeo [18] and Dai [10] all studied the eta invariant under such so-called adiabatic limits. In 1990, Müller linked his work on signature theorems for manifolds with cusps of rank 1 to this new work on the eta invariant [20] and interpreted it in terms of middle perversity intersection cohomology. Dai was able to show from this that the $L^{2}$ signature for such a manifold was equal to the $L^{2}$ signature for the manifold with boundary formed by cutting off the cusps plus the $\tau$ invariant of the resulting boundary fibre bundle, defined in [10].

Recently, Dai and Cheeger followed up Cheeger's Hodge theorem with a signature theorem for conical pseudomanifolds. In [7], they consider the case of a conical pseudomanifold $X$ with one even codimensional smooth singular stratum, $B$. They show that the signature of the intersection pairing on middle degree $L^{2}$ forms on the smooth stratum of $X$ is given by the formula

$$
\sigma(X)=\operatorname{sgn} \operatorname{Im}\left(H^{*}(X, B) \longrightarrow H^{*}\left(X^{\mathrm{reg}}\right)\right)+\tau,
$$

where $\tau$ is the invariant defined in [10] of the fibre bundle over $B$ that forms the boundary of the normal neighborhood of the singular stratum. Recently, in [16], the author and Mazzeo have extended Cheeger and Dai's results to conical pseudomanifolds with one singular stratum in the form of a smooth compact manifold that may be odd dimensional, where the signature theorem turns out to have the same form. The Hodge theorem in this case, however, depends on the ideal boundary condition chosen.

There are clear relationships among the topological objects arising in $L^{2}$ Hodge and signature results for manifolds with boundary and those arising in theorems for manifolds with conical singularities. Specifically, there is an interpolation between the objects arising in theorems for manifolds with boundary and for manifolds with 
conical singularities. The interpolation between the relative and absolute cohomologies of $X^{\text {reg }}$ was first studied by Zeeman in [22]. It was given a geometric interpretation in McCrory's paper on the Zeeman filtration [19] which was extended by Habegger and Saper in [14]. McCrory's work in fact inspired the development of intersection homology groups by Goresky and MacPherson. The dual intersection cohomology groups interpolate between relative and absolute cohomologies as a parameter called the perversity changes.

In the context of Hodge theorems for manifolds with boundary and with conical singularities, these relationships lead to the question of whether the topological interpolation through intersection cohomology groups is related to a natural interpolation in $L^{2}$ cohomologies. That is, can we realize each intermediate intersection cohomology group as we smoothly vary the metric on the end of $X^{\text {reg }}$ between a conical metric and a cone bundle metric? It turns out that we can, which is the content of our first theorem. A version of this question was also studied by Nagase in [21], and we discuss its relationship to the present paper below.

We can ask a similar question about the $L^{2}$ signature theorems for cylindrical and cone bundle metrics on $X^{\text {reg }}$, which differ by a topological invariant, $\tau$. This invariant is defined as a sum of smaller topological invariants, so partial sums of these interpolate naturally between 0 and $\tau$. Thus we can also ask if this topological interpolation is related to an interpolation in metrics. In this paper, we generalize the techniques of [7] to answer this question in the affirmative, as well. This is the content of our second theorem.

Consider a compact manifold $\bar{M}$ with boundary $\partial \bar{M}=Y$ where $Y \stackrel{\phi}{\rightarrow} B$ is a fibre bundle with fibre $F$. Endow $M=\bar{M}-\partial \bar{M}$ with a metric $g_{c}$ that is quasi-isometric near the boundary to a metric of the form

$$
d s_{c}^{2}=d r^{2}+r^{2 c} \tilde{h}+\phi^{*} d s_{B}^{2},
$$

where $\tilde{h}$ is a two-form that restricts to a metric on each fibre of $Y$ and $0 \leq c \leq 1$. Note that these metrics interpolate between a cylindrical metric when $c=0$ and Cheeger's conical metric when $c=1$. In this case, the conical metric gives the manifold the form of a cone bundle over $B$ near the boundary. There is a second natural compactification, $X$, for the manifold $M$, obtained by collapsing the fibres of the boundary fibre bundle and adding a compactifying copy of the base, or, equivalently, adding the cone points to each fibre. The resulting space $X$ is then a conical pseudomanifold as considered by Cheeger.

As mentioned before, on a complete manifold, the exterior derivative $d$ on compactly supported smooth forms and its formal adjoint $\delta= \pm * d *$ each has a unique extension 
to $L^{2}$ forms, and the Hodge Laplacian given by these extensions, $\bar{d}+\bar{\delta}$, is self-adjoint. On an incomplete manifold, this is not generally true. There may be many closed extensions of $d$, and many self-adjoint extensions of the Hodge Laplacian. Perhaps the most natural of these are the minimal and maximal extensions of $d$ and $\delta$ and their associated self-adjoint Laplacians. Recall that the minimal and maximal extensions of the exterior derivative, $d$, on compact smooth forms to $L^{2}$ forms on $\left(M, g_{c}\right)$ are defined by:

Definition 1 Let $\sigma \in L^{2} \Omega^{k}(M, g)$ for any $k$. If there exists an $\eta \in L^{2} \Omega^{k+1}(M, g)$ such that for all $\phi \in C_{0}^{\infty} \Omega^{k+1}(M, g)$ we have

$$
\langle\sigma, \delta \phi\rangle_{L^{2}(M)}=\langle\eta, \phi\rangle_{L^{2}(M)},
$$

we say that $\sigma \in \mathcal{D}\left(d_{\max }\right)$ and we write $d_{\max } \sigma=\eta$.

Definition 2 If $\sigma \in L^{2} \Omega^{k}(M, g)$ for any $k$, and if there is a sequence $\left\{\sigma_{n}\right\} \subset$ $C_{0}^{\infty} \Omega^{k}(M, g)$ such that $\sigma=\lim _{L^{2}} \sigma_{n}$ and $\zeta=\lim _{L^{2}} d \sigma_{n}$, we say $\sigma \in \mathcal{D}\left(d_{\min }\right)$ and $\zeta=d_{\min } \sigma$. In this case, it is always true that $\sigma \in \mathcal{D}\left(d_{\max }\right)$ as well, and $d_{\max } \sigma=d_{\min } \sigma$.

Note that this second extension is the one corresponding to the graph closure of $d: C_{0}^{\infty} \Omega^{*}(M, g) \longrightarrow C_{0}^{\infty} \Omega^{*}(M, g)$ in $L^{2} \Omega^{*}(M, g)$. The minimal and maximal extensions of $\delta$ are defined analogously. Both extensions of $d$ define cohomology groups on $M$, which we will denote by $H_{\min }^{*}\left(M, g_{c}\right)$ and $H_{\max }^{*}\left(M, g_{c}\right)$. The latter is often called simply the $L^{2}$ cohomology. We define two related self-adjoint extensions of the Hodge Laplacian $D=d+\delta$ :

$$
\begin{aligned}
D_{\text {rel }} & =d_{\min }+\delta_{\max } \\
D_{\text {abs }} & =d_{\text {max }}+\delta_{\text {min }} .
\end{aligned}
$$

We will denote the corresponding spaces of harmonic forms by $\mathcal{H}_{\mathrm{rel}}^{*}\left(M, g_{c}\right)$ and $\mathcal{H}_{\text {abs }}^{*}\left(M, g_{c}\right)$, respectively, in reference to the Hodge theorem in the case of a manifold with boundary. Note that in general, however, these spaces of harmonic forms are not isomorphic to relative and absolute cohomology.

Our first theorem is a Hodge theorem for these spaces:

Theorem 1 Let $\bar{M}$ be a compact manifold with boundary $\partial \bar{M}=Y$ where $Y \stackrel{\phi}{\rightarrow} B$ is a fibre bundle with $f$-dimensional fibre $F$. Endow $M=\bar{M}-\partial \bar{M}$ with a metric $g_{c}$ that is quasi-isometric near the boundary to a metric of the form

$$
d s_{c}^{2}=d r^{2}+r^{2 c} \tilde{h}+\phi^{*} d s_{B}^{2}
$$


where $\tilde{h}$ is a two-form that restricts to a metric on each fibre of $Y$. Let $X$ be the compactification of $M$ obtained by collapsing the fibres of the boundary fibre bundle. Then

$$
\begin{array}{r}
H_{\text {min }}^{*}\left(M, g_{c}\right) \cong \mathcal{H}_{\text {rel }}^{*}\left(M, g_{c}\right) \cong \begin{cases}I H_{\frac{\mathfrak{m}-\llbracket \frac{1}{2 c} \rrbracket}{*}(X, B)} & f \text { is even } \\
I H_{\frac{\mathfrak{m}}{*} \llbracket \frac{1}{2}+\frac{1}{2 c} \rrbracket}^{*}(X, B) & f \text { is odd }\end{cases} \\
H_{\text {max }}^{*}\left(M, g_{c}\right) \cong \mathcal{H}_{\mathrm{abs}}^{*}\left(M, g_{c}\right) \cong \begin{cases}I H_{\overline{\mathfrak{m}}+\llbracket \frac{1}{2 c} \rrbracket}^{*}(X, B) & f \text { is even } \\
I H_{\overline{\mathfrak{m}}+\llbracket \frac{1}{2}+\frac{1}{2 c} \rrbracket}^{*}(X, B) & f \text { is odd }\end{cases}
\end{array}
$$

where $\llbracket x \rrbracket$ denotes the greatest integer strictly less than $x$ and $\underline{\mathfrak{m}}$ and $\overline{\mathfrak{m}}$ are the two middle perversities for intersection cohomology on $X$.

Here we use the notation $I H_{\mathfrak{p}}^{*}(X, B)$ instead of $I H_{\mathfrak{p}}^{*}(X)$ in order to indicate a slightly more general definition of intersection cohomology than is standard. In particular, it allows us to include the case where $F$, the fibre of the boundary fibre bundle, is trivial, so $X$ is our original manifold with boundary, $\bar{M}$. Also, in this paper, we use the standard definition of intersection cohomology, rather than the dual definition used in [16] and [15]. Thus in particular, the notations $\mathfrak{m}$ and $\overline{\mathfrak{m}}$ are not the same as in those papers, but their meaning is swapped. We discuss intersection cohomology further in Section 2.1.

Note that when $c=1$, we get $\llbracket \frac{1}{2}+\frac{1}{2 c} \rrbracket=0$, so this result reduces to the result for manifolds with cone bundle ends (called incomplete edges in [16]), and in the case that the fibre is even dimensional, it reduces to Cheeger's result in [6]. In the extended definition of intersection cohomology, for $c$ sufficiently close to 0 , the minimal and maximal $L^{2}$ cohomologies for $\left(M, g_{c}\right)$ become relative and absolute cohomologies of $M$, respectively, thus reducing to Conner's results [9] for manifolds with boundary. As $c$ goes from 0 to 1 , the intersection cohomology groups isomorphic to the maximal cohomology interpolate between upper middle perversity and absolute cohomology, while the intersection cohomology groups isomorphic to the minimal cohomology interpolate between lower middle perversity and relative cohomology. There is substantial overlap between this result in the case of maximal cohomology and the work of Nagase in [21], in which he constructs metrics to realize various perversity intersection cohomologies of Witt spaces as (maximal) $L^{2}$-cohomologies. Certainly the maximal cohomology result in the case that $f$ is odd is contained in Nagase's work, and it seems likely that with attention to the question of ideal boundary conditions, his paper could generalize to give the entire Hodge theorem above, and, indeed, a more general one. 
Using our Hodge theorem, we can also obtain a signature theorem for the manifolds $\left(M, g_{c}\right)$ through a signature theorem for intersection cohomology. If $p$ and $q$ are dual perversities with $p \leq q$, then we can define an intersection form on spaces of the form

$$
\operatorname{Im}\left(I H_{p}^{n / 2}(X, B) \longrightarrow I H_{q}^{n / 2}(X, B)\right)
$$

as we will discuss in Section 4.1. We will call the signature of such an intersection form a perverse signature, and denote it by $\sigma_{p}(X)$.

The $\tau$-invariant defined by Dai in [10] is given by a sum $\tau=\sum_{i=2}^{\infty} \tau_{i}$, where $\tau_{i}$ is the signature of a form defined on the $E_{i}$ term of the Leray-Serre spectral sequence for the boundary fibre bundle of $M$. Our signature theorem for metrics interpolating between finite cylindrical and conical is:

Theorem 2 If $p=\underline{\mathfrak{m}}+k$, then the signature of the intersection form on these spaces is given by

$$
\sigma_{p}(X)=\operatorname{sgn} \operatorname{Im}\left(H^{*}(M, \partial M) \longrightarrow H^{*}(M)\right)+\sum_{i=2+2 k}^{\infty} \tau_{i} .
$$

Thus as the metric becomes less and less cylindrical and more and more conical, the signature theorem picks up more and more of the $\tau_{i}$ terms, until, when the metric is close to conical, the signature includes all of $\tau$. This theorem allows us to interpret the summands, $\tau_{i}$, as differences between perverse signatures for adjacent perversities.

It seems likely that the theorems in this paper should generalize. For instance, by relying more on strictly topological methods and using the basic definitions and results about intersection cohomology, it should be possible to define a signature pairing for any perversity intersection cohomology on any pseudomanifold and to obtain a more general version of Novikov additivity for their perverse signatures. In a different direction, the author is currently working with Daniel Grieser on similar interpolating Hodge and signature theorems for a family of complete metrics on $M$ that interpolates between the fibred cusp metric results in [15] and the noncompact cylindrical metric results of [1].

The remainder of this paper is organized as follows. In Section 2, we give definitions and background on intersection cohomology, the Leray-Serre spectral sequence and Hilbert complexes, all of which are used in the proofs of the main theorems. In Section 3 we prove the Hodge theorem by reducing to a local calculation near the singular stratum as in [6] and [16]. In Section 4 we define all of the versions of perverse signatures we will use and prove a version of Novikov additivity for them. Finally we prove the 
signature theorem through a careful analysis of the Leray-Serre spectral sequence of the boundary fibre bundle. Our techniques throughout the paper are direct generalizations of the techniques of [7].

The author acknowledges partial support by the NSF through an ROA supplement to grant DMS-0204730. The author would also like to thank Tamás Hausel, Rafe Mazzeo and especially Xianzhe Dai for useful conversations relating to this paper, and the referee, whose careful comments greatly improved its exposition.

\section{Background}

Before we begin the proof of Theorem 1, we will briefly review some definitions and theorems we will use in our proofs.

\subsection{Intersection cohomology}

Intersection cohomologies are topological invariants defined on pseudomanifolds. An $n-$ dimensional pseudomanifold is a paracompact Hausdorff space $X$ with a stratification,

$$
X_{0} \subset X_{1} \subset \cdots \subset X_{n-1} \subset X_{n}=X
$$

such that for any point $x$ on the codimension $\ell$ stratum, $X_{n-\ell} \backslash X_{n-\ell-1}$, there is a neighborhood of $x$ in $X$ homeomorphic to the product of a ball $\Delta \subset \mathbb{R}^{n-\ell}$ with the cone, $C\left(L_{\ell}\right)$, on a lower dimensional pseudomanifold, $L_{\ell}$, called the link. In the standard definition, a pseudomanifold is also required to have no $n-1$ stratum, that is, the codimension two stratum must be dense in $X$. We will relax that requirement in this paper as we discuss below.

Intersection cohomologies for a given pseudomanifold are parametrized by a function called the perversity function, $\mathfrak{p}:\{1,2, \ldots, n\} \longrightarrow \mathbb{Z}$, that assigns to the stratum of codimension $\ell$ the integer $\mathfrak{p}(\ell)$. The perversity function must satisfy the properties that $\mathfrak{p}(0)=\mathfrak{p}(1)=\mathfrak{p}(2)=0$ and $\mathfrak{p}(\ell) \leq \mathfrak{p}(\ell+1) \leq \mathfrak{p}(\ell)+1$. We will also relax these conditions in this paper.

We will prove $H_{\max }^{*}\left(M, g_{c}\right)$ is isomorphic to an intersection cohomology group via a sheaf argument. For a complex of sheaves $\left(\mathcal{L}^{*}, d\right)$ on a pseudomanifold $W$, let $H^{*}(W, \mathcal{L})$ denote the cohomology of its global sections. Then we have the following theorem of Goresky and MacPherson:

Proposition 1 [12] Let $X$ be a pseudomanifold and let $\left(\mathcal{L}^{*}, d\right)$ be a complex of fine sheaves on $X$. Assume that there is a map from the constant sheaf to $\mathcal{L}^{*}$ that induces 
local isomorphisms on cohomology over the top (smooth) stratum of $X$. Assume also that for any point $x$ on the codimension $\ell$ stratum, $S$, of $X$ with neighborhood $U \cong \Delta \times C\left(L_{\ell}\right)$, the local cohomology $H^{i}(U, \mathcal{L})=0$ for $i>\mathfrak{p}(\ell)$. Finally, assume that $H^{i}(U, \mathcal{L}) \cong H^{i}(U \backslash(U \cap S), \mathcal{L})$ for $i \leq p(\ell)$ under the natural attaching map. Then $H^{*}(X, \mathcal{L}) \cong I H_{\mathfrak{p}}^{*}(X)$, the intersection cohomology of $X$ of perversity $\mathfrak{p}$.

In the case of $L^{2}$ cohomology, the natural attaching map is given by restriction. This means that to show that $L^{2}$ cohomology is isomorphic to intersection cohomology of some perversity, we need to construct a fine sheaf of $L^{2}$ forms and show that, in the neighborhood $U \cong \Delta \times C\left(L_{l}\right)$ of a point $x$ on the codimension $\ell$ stratum, it satisfies the recursive local calculation for intersection cohomology:

$$
H^{i}(\mathcal{U}, \mathcal{L}) \cong I H_{\mathfrak{p}}^{i}(\mathcal{U})= \begin{cases}I H_{\mathfrak{p}}^{i}(L) & i \leq \mathfrak{p}(\ell) \\ 0 & i>\mathfrak{p}(\ell)\end{cases}
$$

In this paper, the singular space we will associate to a manifold with fibre bundle boundary is the space $X$ formed by coning off the fibres at the boundary. This will be a stratified space with one singular stratum that is smooth and homeomorphic to the base, $B$, of the boundary fibre bundle. If the fibre $F$ is nontrivial, then it will be the link of this stratum, and $X$ will be a pseudomanifold as described above. In this case, the local calculation for intersection cohomology in a neighborhood of a point $b$ on the singular stratum $\mathcal{U} \cong \Delta \times C(F)$ for $b \in \Delta$ a disk in $B$ is

$$
H^{i}(\mathcal{U}, \mathcal{L}) \cong I H_{\mathfrak{p}}^{i}(\mathcal{U})= \begin{cases}H^{i}(F) & i \leq p, \\ 0 & i>p\end{cases}
$$

where the perversity function $\mathfrak{p}$ takes the value, $p=\mathfrak{p}(f+1)$, on the only relevant stratum, namely the singular one.

Note that for larger values of $\mathfrak{p}$, the truncation in $H^{i}(F)$ occurs at a higher degree. Thus if $\mathfrak{p}<\mathfrak{q}$, then $I H_{\mathfrak{p}}^{i}(U)$ vanishes for more degrees than $I H_{\mathfrak{q}}^{i}(U)$. In the extremes, if $H^{i}(\mathcal{U}, \mathcal{L}) \cong H^{i}(F)$ for all $i$, this is the local calculation for absolute cohomology of $X^{\text {reg }}$. If $H^{i}(\mathcal{U}, \mathcal{L})=\{0\}$ for all $i$, then this is the local calculation for the cohomology of $X$ relative to its singular stratum. Thus we can generalize this definition slightly as follows:

$$
I H_{p}^{*}(X, B)= \begin{cases}H^{*}(X, B) & p \leq-1, \\ I H_{\mathfrak{p}}^{*}(X) & 0 \leq p \leq f-1, \\ H^{*}(X \backslash B) & p \geq f .\end{cases}
$$

By doing this, we are also able to include the case where $F$ is a point, and $X$ is simply a manifold with boundary. Since our proofs will use only the local calculations, we are thus able to treat both cases simultaneously. The only property of intersection 
cohomology that is lost by this generalization is the invariance of the definition under a change of stratification. As we use a specific stratification of $X$ in our theorems, this does not cause any ambiguity.

Importantly, the Poincaré duality property still does hold under this extension of the definition. It can be stated in terms of two particular perversities on a pseudomanifold, called the middle perversities: $\overline{\mathfrak{m}}(\ell)=\lceil(\ell-2) / 2\rceil$ and $\mathfrak{m}(\ell)=\lceil(\ell-3) / 2\rceil$, where $\lceil x\rceil$ denotes the least integer greater than or equal to $x$. In the case of a pseudomanifold with a single smooth singular stratum, the local calculations near a point on the compactifying layer $B$ for the two middle perversities are

$$
\begin{aligned}
H^{i}(\mathcal{U}, \mathcal{L}) & \cong I H_{\underline{\mathfrak{m}}}^{i}(\mathcal{U}) \\
& = \begin{cases}H^{i}(F) & i \leq \frac{f}{2}-1 \text { if } f \text { is even and } i \leq \frac{f-1}{2} \text { if } f \text { is odd } \\
0 & i \geq \frac{f}{2} \text { if } f \text { is even and } i \geq \frac{f+1}{2} \text { if } f \text { is odd }\end{cases}
\end{aligned}
$$

for the lower middle perversity and

$$
\begin{aligned}
H^{i}(\mathcal{U}, \mathcal{L}) & \cong I H \frac{i}{\mathfrak{m}}(\mathcal{U}) \\
& = \begin{cases}H^{i}(F) & i \leq \frac{f}{2} \text { if } f \text { is even and } i \leq \frac{f-1}{2} \text { if } f \text { is odd } \\
0 & i \geq \frac{f}{2}+1 \text { if } f \text { is even and } i \geq \frac{f+1}{2} \text { if } f \text { is odd }\end{cases}
\end{aligned}
$$

for the upper middle perversity. Note that in the simplest case, where the fibre is a point, these local calculations correspond to $H_{0}^{*}(M)$ and $H^{*}(M)$, respectively.

In the case that the strata of $X$ are all even dimensional, $X$ is a particular kind of pseudomanifold called a Witt space. In this case, the two middle perversity intersection cohomologies are identical, and this single middle perversity intersection cohomology satisfies Poincaré duality. If the strata are not all even dimensional, the two middle perversities are not generally the same. In this case, they are Poincaré duals of each other. That is to say, there is a nondegenerate pairing between the spaces. In general, every perversity $\mathfrak{p}$ has a dual perversity $\mathfrak{q}=\mathfrak{t}-\mathfrak{p}$ where $\mathfrak{t}(\ell)=\ell-2$ is called the total perversity. That is, the intersection cohomologies for perversities $\mathfrak{p}$ and $\mathfrak{q}$ are Poincaré duals. In the case of our stratified spaces with only one singular stratum, this means perversities that lie at equal distances from the two middle perversities, $\overline{\mathfrak{m}}+k$ and $\underline{\mathfrak{m}}-k$, are also dual perversities. Note that in the case of a trivial fibre, this duality is then the well known duality between relative and absolute cohomologies.

\subsection{The Leray-Serre spectral sequence and the geometry of fibre bundles}

We will use a careful analysis of the Leray-Serre spectral sequence of the boundary fibre bundle to prove the signature theorem for our family of metrics. For this analysis, 
it is useful to think of elements arising in the Leray-Serre spectral sequence of a fibre bundle in terms of bidegree. Recall from geometry of fibre bundles that we can define the bidegree of a form on the total space of a fibre bundle as follows. Let $\phi: Y \rightarrow B$ be a fibre bundle with fibre $F$, and suppose that it is endowed with a metric $G$ of the form $\phi^{*}(h)+k$, where $h$ is a metric on $B$. Assume further that $\phi:(Y, G) \rightarrow(B, h)$ is a Riemannian submersion. The tangent bundle $T Y$ splits into a vertical and horizontal subbundle, $T^{V} Y \oplus T^{H} Y$, where $T^{V} Y=\operatorname{ker}(d \phi)$ and $T^{H} Y$ is its orthogonal complement (and is also the subbundle annihilated by $k$ ). This induces a splitting of the form bundles on $Y$, and thus every differential form has a (horizontal,vertical) bidegree, ie

$$
\Omega^{p, q}(Y)=\Omega^{p}(B) \widehat{\otimes} \Omega^{q}\left(Y, T^{V} Y\right) .
$$

We will refer to the horizontal and vertical degrees as base and fibre degrees, respectively, as in the product bundle case, although strictly speaking this is not accurate more generally. In terms of this decomposition of forms, we can also decompose the exterior derivative operator. For a full treatment of this decomposition, see Hausel, Hunsicker and Mazzeo [15]. For this paper, it is relevant only to note that the exterior derivative operator on $Y$ always preserves or increases the base degree of a form, and the part of the operator that does not increase the base degree is exactly the exterior derivative operator on the fibres, $d_{F}$. Thus the complex of forms on the boundary, $Y$, forms a filtered differential graded module (vector space, in fact) with grading by form degree and filtration by minimum base degree. This is precisely the grading and filtration used to construct the Leray-Serre spectral sequence for the cohomology of $Y$. On

the 0 -th page of this spectral sequence, $E_{0}^{p, q} \cong \Omega^{p, q}(Y)$. A representative of a class is a $(p, q)$ bidegree form plus any form of higher base and lower fibre degree. The differential $d_{0}$ is given by $d_{F}$ on the $(p, q)$ part of the form. On the first page, $E_{1}^{p, q} \cong \Omega^{p}\left(B, H^{*}(F)\right)$, so a representative of a class is a fibre harmonic $(p, q)$ form plus any fibre exact $(p, q)$ form plus any form of higher base and lower fibre degree. On the second page, $E_{2}^{p, q} \cong H^{p}\left(B, H^{q}(F)\right)$. Because the overall differential, $d$, respects the filtration, note that on all pages, $E_{r}^{*, *}$ of the spectral sequence, a representative of a class in $E_{r}^{p, q}$ will be the sum of a "leading" term of bidegree $(p, q)$ and additional terms of strictly higher base and lower fibre degree. When we consider the intersection pairing in terms of this spectral sequence, we will see that only the leading terms contribute in the end to the signature.

\subsection{The $\tau$-invariant}

The $\tau$-invariant was introduced by Dai in [10]. It can be interpreted as characterizing the nonmultiplicativity of signature for manifolds with fibration boundary. It is defined 
in terms of the Leray-Serre spectral sequence of the boundary fibration. We repeat the definition from [10] here for reference. Let $Y$ be the total space of a fibre bundle with oriented fibre of dimension $f$ and oriented base of dimension $b$. Assume that $\operatorname{dim}(Y)=f+b=4 k-1$ for some integer $k$. Let

$$
\left(E_{r}^{i, j}, d_{r}\right)_{\substack{i=1, \ldots, b \\ j=1, \ldots, f}}
$$

$r \geq 2$, denote the $r$-th page of the Leray-Serre spectral sequence for $Y$. Let $E_{r}^{s}=$ $\sum_{i+j=s} E_{r}^{i, j}$. There is a basis $\beta_{r}$ for each $E_{r}^{4 k-1}$ induced from the orientation on $Y$. For each $p+q=4 k-2$, define a pairing on each page of the spectral sequence by

$$
\begin{aligned}
\sigma_{r}: E_{r}^{p} \otimes E_{r}^{q} & \longrightarrow \mathbb{R}, \\
& \phi \otimes \psi
\end{aligned}
$$

When $p=q=2 k-1$, this pairing is symmetric. We then denote its signature by $\tau_{r}$, and we define $\tau=\sum_{r \geq 2} \tau_{r}$.

On each page of the Leray-Serre spectral sequence, we can decompose the pairing above in terms of bidegree. In terms of this decomposition, it turns out that only one term contributes to the signature, $\tau_{r}$, namely, the one corresponding to the pairing $E_{r}^{(b-r) / 2,(f+r-1) / 2} \otimes E_{r}^{(b-r) / 2,(f+r-1) / 2}$. This is proved in [7], and we recap the proof in the context of the proof of Lemma 5 in Section 4 of this paper. One consequence is that only $\tau_{r}$ for even $r$ contribute to $\tau$ when the fibre is odd dimensional, and only $\tau_{r}$ for odd $r$ contribute to $\tau$ when the fibre is even dimensional.

\subsection{Hilbert complexes}

There are a number of basic results we will use in the proof of our Hodge theorem that are true in great generality. They have been proved for objects called Hilbert complexes in [4], and we summarize them here for reference.

A Hilbert complex is a complex, $\left(L_{*}, D_{*}\right)$, of the form

$$
0 \rightarrow L_{0} \stackrel{D_{0}}{\longrightarrow} L_{1} \stackrel{D_{1}}{\longrightarrow} L_{2} \ldots \stackrel{D_{n-1}}{\longrightarrow} L_{n} \rightarrow 0
$$

where each $L_{i}$ is a separable Hilbert space, the differentials $D_{i}: L_{i} \rightarrow L_{i+1}$ are closed operators with dense domain $\mathcal{D}\left(D_{i}\right)$ such that $\operatorname{ran}\left(D_{i}\right) \subseteq \mathcal{D}\left(D_{i+1}\right)$ and $D_{i+1} \circ D_{i}=0$ for all $i$. Given such a complex there is a dual Hilbert complex

$$
0 \longleftarrow L_{0} \stackrel{D_{0}^{*}}{\longleftarrow} L_{1} \stackrel{D_{1}^{*}}{\longleftarrow} L_{2} \ldots \stackrel{D_{n-1}^{*}}{\longleftarrow} L_{n} \longleftarrow 0
$$


defined using the Hilbert space adjoints of the differentials, $D_{i}^{*}: L_{i+1} \rightarrow L_{i}$, and a Laplacian $\Delta_{i}=D_{i}^{*} D_{i}+D_{i-1} D_{i-1}^{*}$. This is a self-adjoint operator on $L_{i}$ with domain

$$
\mathcal{D}\left(\Delta_{i}\right)=\left\{u \in \mathcal{D}\left(D_{i}\right) \cap \mathcal{D}\left(D_{i-1}^{*}\right): D_{i} u \in \mathcal{D}\left(D_{i}^{*}\right), D_{i-1}^{*} u \in \mathcal{D}\left(D_{i-1}\right)\right\}
$$

and nullspace $\quad \operatorname{ker} \Delta_{i}:=\mathcal{H}^{i}\left(L_{*}, D_{*}\right)=\operatorname{ker} D_{i} \cap \operatorname{ker} D_{i-1}^{*}$.

We get many of the standard results for these operators. First, there is a weak Kodaira decomposition:

Lemma 1 [4, Lemma 2.1] If $\left(L_{*}, D_{*}\right)$ is a Hilbert complex and $\left(L_{*}, D_{*}^{*}\right)$ is its adjoint, then

$$
L_{i}=\mathcal{H}^{i} \oplus \overline{\operatorname{ran} D_{i-1}} \oplus \overline{\operatorname{ran} D_{i}^{*}}
$$

Second, the cohomology of the complex, defined by

$$
H^{i}\left(L_{*}, D_{*}\right)=\operatorname{ker} D_{i} / \operatorname{ran} D_{i-1}
$$

and cohomology of the dual complex are either both finite dimensional or both infinite dimensional [4, Corollary 2.6]. In the finite dimensional case, we have:

Lemma 2 [4, Corollary 2.5] If the cohomology of the complex is finite dimensional, then $\operatorname{ran}\left(D_{i-1}\right)$ is closed and $H^{i}\left(L_{*}, D_{*}\right)=\mathcal{H}^{i}\left(L_{*}, D_{*}\right)$.

There is a Künneth theorem:

Proposition 2 [4, Corollary 2.15] Let $\left(L^{\prime}, D^{\prime}\right)$ and $\left(L^{\prime \prime}, D^{\prime \prime}\right)$ be two Hilbert complexes. Form the completed tensor product Hilbert complex $(L, D)$ where

$$
\begin{gathered}
L_{j}=\bigoplus_{i+\ell=j} L_{i}^{\prime} \hat{\otimes} L_{\ell}^{\prime \prime}, \\
D_{j}=\bigoplus_{i+\ell=j}\left(D_{i}^{\prime} \otimes \mathrm{id}_{L_{\ell}^{\prime \prime}}+(-1)^{i} \mathrm{id}_{L_{i}^{\prime}} \otimes D_{\ell}^{\prime \prime}\right) .
\end{gathered}
$$

Suppose that $D^{\prime \prime}$ has closed range in all degrees. Then

$$
H^{j}(L, D)=\bigoplus_{i+\ell=j} H^{i}\left(L^{\prime}, D^{\prime}\right) \otimes H^{\ell}\left(L^{\prime \prime}, D^{\prime \prime}\right)
$$


Finally, we may compute these cohomology groups using a "core subcomplex"

$$
\mathcal{D}^{\infty} L_{i} \subset L_{i}
$$

for all $i$ consisting of all elements $\omega$ that are in the domain of $\Delta_{i}^{\ell}$ for all $\ell \geq 0$.

Proposition 3 [4, Theorem 2.12] The cohomology of the complex $\left(L_{*}, D_{*}\right)$ is equal to the cohomology of the complex $\left(\mathcal{D}^{\infty} L_{*}, D_{*}\right)$.

In the case of the maximal and minimal $L^{2}$ cohomologies for a manifold with boundary (or with singularities), this implies that $H_{\text {max }}^{*}(M, g)$ and $H_{\text {min }}^{*}(M, g)$ can be computed using the complex of forms that are smooth on the interior (or smooth set) of $(M, g)$.

\section{Proof of Hodge theorem}

As mentioned before, we will prove this theorem using Proposition 1. The proof will have three steps. In the first step, we reduce the proof to establishing a Poincaré lemma, in the second step we prove some preliminary results, and in the third step we prove the Poincaré lemma.

\subsection{Reduction to Poincaré lemma}

The two complexes $\left(D\left(d_{\max }\right), d_{\max }\right)$ and $\left(D\left(d_{\min }\right), d_{\min }\right)$ form Hilbert complexes as defined in Brüning and Lesch [4], so we can use the theory developed in that paper to prove Theorem 1. First we note that it suffices to prove the theorem for $\left(D\left(d_{\max }\right), d_{\max }\right)$. The Hodge star operator gives a isomorphism of complexes between this complex and the dual complex $\left(D\left(d_{\min }\right), d_{\min }\right)$, so the maximal complex has finite dimensional cohomology if and only if the minimal complex does. Furthermore, these two are Poincaré dual spaces, so if $H_{\text {max }}^{*} \cong I H_{\mathfrak{p}}^{*}(X, B)$, then we must also have $H_{\text {min }}^{*} \cong I H_{\mathfrak{q}}^{*}(X, B)$, where $\mathfrak{p}$ and $\mathfrak{q}$ are dual perversities.

Finally, if these cohomologies are finite dimensional, for instance, if they are isomorphic to intersection cohomologies, then by [4, Corollary 2.5], we immediately get the isomorphisms

and

$$
H_{\min }^{*}\left(M, g_{c}\right) \cong \mathcal{H}_{\mathrm{rel}}^{*}\left(M, g_{c}\right)
$$

$$
H_{\max }^{*}\left(M, g_{c}\right) \cong \mathcal{H}_{\mathrm{abs}}^{*}\left(M, g_{c}\right) .
$$

Thus it suffices to show that $H_{\max }^{*}\left(M, g_{c}\right) \cong I H_{\overline{\mathfrak{m}}+k}(X, B)$, where $k=\llbracket \frac{1}{2 c} \rrbracket$ if $f$ is even and $\left[\left[\frac{1}{2}+\frac{1}{2 c}\right]\right]$ if $\mathrm{f}$ is odd. 
We can create a complex of fine sheaves of $L^{2}$ forms on $X$ in the same manner as in the proofs of [15, Proposition 2] and [23, Proposition 1.18]. By Proposition 1, we need only then prove a local Poincaré lemma to establish the isomorphism between $H_{\max }^{*}\left(M, g_{c}\right)$ and intersection cohomology of some perversity. The local Poincaré lemma for points on the stratum $M \subset X$ is identical to the standard Poincare lemma for compact manifolds. Near a point on the singular stratum, we need to establish a Poincare lemma for neighborhoods of the form $U=\Delta \times C(F)$, where $\Delta$ is a disk in the singular stratum. By the Künneth Theorem [4, Corollary 2.15] this calculation reduces to the calculation on a simple cone by

$$
H_{\max }^{*}\left(U, g_{c}\right) \cong H_{\max }^{*}(\Delta) \otimes H_{\max }^{*}\left(C(F), g_{c}\right) \cong H_{\max }^{*}\left(C(F), g_{c}\right),
$$

so the necessary Poincare lemma is one for the cone on $F$ with respect to the metric $g_{c}=d r^{2}+r^{2 c} d s_{F}^{2}$.

\subsection{Preliminary propositions}

The proof of the necessary Poincaré lemma is based on the techniques in Cheeger [6]. Before proceeding to the proof, we'll lay out a few facts we will use. First, by [4, Theorem 2.12], we know that the natural inclusion of smooth $L^{2}$ forms into the space of $L^{2}$ forms induces an isomorphism on cohomology for both the maximal and minimal complexes, so we may always assume a maximal cohomology class has a smooth representative. This is true for any smooth manifold. Next we prove some propositions specific to the metrics $g_{c}$ on cones.

Proposition 4 Let $\phi \neq 0$ be an $i$-form on $F$. Then the pullback of $\phi$ to $C(F)$ is in $L^{2} \Omega^{i}\left(C(F), g_{c}\right)$ if and only if $i<\frac{f}{2}+\frac{1}{2 c}$, and in this case, the pullback map is bounded.

Proof If $\phi$ is an $i$-form on $F$, then

$$
\int_{C(F)}\|\phi\|_{c}^{2} d \operatorname{vol}_{c}=\|\phi\|_{L^{2}(F)}^{2} \int_{0}^{1} r^{c(f-2 i)} d r<\infty
$$

if and only if $c(f-2 i)>-1$, which is true if and only if $i<\frac{f}{2}+\frac{1}{2 c}$. Since the integral on the right is independent of $\phi$, the pullback map is bounded.

Proposition 5 There exists a $K>0$ such that for all $\alpha=\phi+d r \wedge \omega \in L^{2} \Omega^{i}\left(C(F), g_{c}\right)$ and for any null set $S \subset(1 / 2,1)$ there is an $a \in(1 / 2,1)-S$ such that

$$
\|\phi(a)\|_{L^{2}(F)}^{2} \leq K\|\phi\|_{L^{2}\left(C(F), g_{c}\right)}^{2} \leq K\|\alpha\|_{L^{2}\left(C(F), g_{c}\right)}^{2} .
$$


Proof Suppose not. Then for any $K>0$, there is a form, $\phi \in L^{2} \Omega^{i}\left(C(F), g_{c}\right)$ such that

$$
\begin{aligned}
\|\phi\|_{L^{2}\left(C(F), g_{c}\right)}^{2} \geq \int_{1 / 2}^{1} \int_{F}\|\phi(r)\|_{F}^{2} r^{-2 i c} d \operatorname{vol}_{F} r^{c f} d r & \\
=\int_{1 / 2}^{1}\|\phi(r)\|_{L^{2}(F)}^{2} r^{c(f-2 i)} d r & >K\|\phi\|_{L^{2}\left(C(F), g_{c}\right)}^{2} \int_{(1 / 2,1)-S} r^{c(f-2 i)} d r \\
& =K\|\phi\|_{L^{2}\left(C(F), g_{c}\right)}^{2} \int_{1 / 2}^{1} r^{c(f-2 i)} d r .
\end{aligned}
$$

So choose $K>\left(\int_{1 / 2}^{1} r^{c(f-2 i)} d r\right)^{-1}$ and we have a contradiction.

Proposition 6 If $i<\frac{f}{2}+\frac{1}{2 c}+1$ and $\alpha=\phi+d r \wedge \omega \in L^{2} \Omega^{i}\left(C(F), g_{c}\right)$, then for any $a \in(1 / 2,1)$,

$$
K_{a}(\alpha)=\int_{a}^{r} \omega(s) d s \in L^{2} \Omega^{i-1}\left(C(F), g_{c}\right)
$$

and $K_{a}$ is a bounded operator, uniformly in $a \in(1 / 2,1)$.

Proof This proposition follows essentially from Schwartz's inequality. By definition,

$$
\left\|K_{a}(\alpha)\right\|_{L^{2}\left(C(F), g_{c}\right)}^{2}=\int_{0}^{1} \int_{F}\left\|\int_{a}^{r} \omega(s) d s\right\|_{F}^{2}{ }^{c}{ }^{c(f-2 i+2)} d \operatorname{vol}_{F} d r,
$$

since $\omega$ is a family of $i-1$ forms on $F$. Consider just the inside of the right hand side of (16):

$$
\left\|\int_{a}^{r} \omega(s) d s\right\|_{F}^{2} \leq\left(\int_{a}^{r}\|\omega(s)\|_{F}\right)^{2}
$$

For any $j$, by the Schwartz inequality, the right side of this is

$$
\begin{aligned}
\left(\int_{a}^{r} s^{-j}\left\|s^{j} \omega(s)\right\|_{F} d s\right)^{2} & \leq \int_{a}^{r} s^{-2 j} d s \int_{a}^{r}\left\|s^{j} \omega(s)\right\|_{F}^{2} d s \\
& = \begin{cases}\frac{r^{1-2 j}-a^{1-2 j}}{1-2 j} \int_{a}^{r}\left\|s^{j} \omega(s)\right\|_{F}^{2} d s \quad j \neq 1 / 2, \\
(\ln (r)-\ln (a)) \int_{a}^{r}\left\|s^{j} \omega(s)\right\|_{F}^{2} d s \quad j=1 / 2 .\end{cases}
\end{aligned}
$$


Choose $2 j=c(f-2 i+2)$ and replace this expression in equation (16) to get:

$$
\begin{aligned}
\left\|K_{a}(\alpha)\right\|_{L^{2}\left(C(F), g_{c}\right)}^{2} \leq & \left\{\begin{array}{c}
\int_{0}^{1} \frac{r-a(r / a)^{c(f-2 i+2)}}{1-c(f-2 i+2)} \int_{a}^{r}\|\omega(s)\|_{F}^{2} s^{c(f-2 i+2)} d s d \operatorname{vol}_{F} d r \\
i \neq \frac{f}{2}+1-\frac{1}{2 c} \\
\int_{0}^{1} r(\ln (r)-\ln (a)) \int_{a}^{r}\|\omega(s)\|_{F}^{2} s^{c(f-2 i+2)} d s d \operatorname{vol}_{F} d r \\
i=\frac{f}{2}+1-\frac{1}{2 c}
\end{array}\right. \\
& \leq\left\{\begin{array}{cc}
\int_{0}^{1} \frac{r-a(r / a)^{c(f-2 i+2)}}{1-c(f-2 i+2)} d r\|\omega\|_{L^{2}\left(C(F), g_{c}\right)}^{2} & i \neq \frac{f}{2}+1-\frac{1}{2 c} \\
\int_{0}^{1} r(\ln (r)-\ln (a)) d r\|\omega\|_{L^{2}\left(C(F), g_{c}\right)}^{2} & i=\frac{f}{2}+1-\frac{1}{2 c}
\end{array}\right.
\end{aligned}
$$

Since $a \in(1 / 2,1)$, the first integral is uniformly bounded in $a$ for $i<\frac{f}{2}+\frac{1}{2 c}+1$ and the second integral is also bounded uniformly in $a$. Thus $K_{a}$ is a bounded operator. $\square$

Proposition 7 Let $\rho>0$, and endow $(\rho, 1) \times F$ with the metric $g_{c}$ restricted from $C(F)$. Let $\alpha=\phi+d r \wedge \omega \in L^{2} \Omega^{i}\left(C(F), g_{c}\right)$. If $i \geq \frac{f}{2}+\frac{1}{2 c}$, then there exists a sequence $\epsilon_{s} \rightarrow 0$ such that

$$
\lim _{\epsilon_{s} \rightarrow 0}\left\|\phi\left(\epsilon_{s}\right)\right\|_{L^{2}\left((\rho, 1) \times F, g_{c}\right)}^{2}=0 .
$$

Proof Since $\alpha \in L^{2} \Omega^{i}\left(C(F), g_{c}\right)$, so is $\phi$, so we know that

$$
\int_{0}^{1} \int_{F}\|\phi(r)\|_{F}^{2} d \operatorname{vol}_{F} r^{c(f-2 i)} d r<\infty .
$$

That is, $\quad \int_{F}\|\phi(r)\|_{F}^{2} d \operatorname{vol}_{F} r^{c(f-2 i)} \in L^{1}[(0,1)]$.

Thus by [6, Lemma 1.2], there is a sequence $\epsilon_{s} \rightarrow 0$ for which

$$
\left|\int_{F}\left\|\phi\left(\epsilon_{S}\right)\right\|_{F}^{2} d \operatorname{vol}_{F} \epsilon_{s}^{c(f-2 i)}\right|<\frac{C}{\epsilon_{S}\left|\ln \left(\epsilon_{S}\right)\right|}
$$

for some constant $C>0$. So we have

$$
\left|\int_{F}\left\|\phi\left(\epsilon_{S}\right)\right\|_{F}^{2} d \operatorname{vol}_{F}\right|<\frac{C \epsilon_{S}^{c(2 i-f)-1}}{\left|\ln \left(\epsilon_{S}\right)\right|} .
$$


Since $i \geq \frac{f}{2}+\frac{1}{2 c}$, the right hand side tends to zero as $\epsilon_{s} \rightarrow 0$. Thus

$$
\begin{aligned}
\left\|\phi\left(\epsilon_{S}\right)\right\|_{L^{2}\left((\rho, 1) \times F, g_{c}\right)}^{2} & =\int_{\rho}^{1} \int_{F}\left\|\phi\left(\epsilon_{S}\right)\right\|_{F}^{2} r^{c(f-2 i)} d \operatorname{vol}_{F} d r \\
& =\left\|\phi\left(\epsilon_{S}\right)\right\|_{L^{2}(F)}^{2} \int_{\rho}^{1} r^{c(f-2 i)} d r \longrightarrow 0
\end{aligned}
$$

also as $\epsilon_{s} \rightarrow 0$.

\subsection{Poincaré lemma}

The Poincaré lemma we need to prove for Theorem 1 is the following:

Lemma 3 Let $(F, g)$ be an $f$-dimensional compact manifold and consider the family of metrics on the cone over $F, C(F)$, given by $d s_{c}^{2}=d r^{2}+r^{2 c} d s_{F}^{2}$ for $0<c<1$. Then

$$
H_{\max }^{i}\left(C(F), g_{c}\right) \cong \begin{cases}H^{i}(F) & i<\frac{f}{2}+\frac{1}{2 c} \\ 0 & i \geq \frac{f}{2}+\frac{1}{2 c}\end{cases}
$$

Proof Consider first the case where $i<\frac{f}{2}+\frac{1}{2 c}$. We want to create a bijective bounded linear map

$$
R: H_{\max }^{i}\left(C(F), g_{c}\right) \longrightarrow H^{i}(F) .
$$

If $\alpha=\phi+d r \wedge \omega \in L^{2} \Omega^{i}\left(C(F), g_{c}\right)$, then for all but a null set of points in $(1 / 2,1)$, we get $\phi \in L^{2}(F)$. So let $[\alpha] \in H_{\max }^{i}\left(C(F), g_{c}\right)$ and for any such value, $a$, define $R([\alpha])=$ $[\phi(a)]$. We need to check that this map makes sense as a map to cohomology rather than just to the space of $i$ forms, that it is independent of our choice of cohomology representative and of $a$, that it is linear, bijective, and that it is bounded with respect to the natural norm on cohomology:

$$
\|[\alpha]\|_{L^{2} H}=\min _{\gamma \in[\alpha]}\|\gamma\|_{L^{2}} .
$$

First, the map clearly makes sense, since if $\alpha$ is closed, then $d \alpha=d_{F} \phi+d r \wedge\left(\phi^{\prime}-\right.$ $\left.d_{N} \omega\right)=0$, thus $d_{F} \phi=0$ for all $a$. To show that the map is well-defined, suppose that $\alpha=\phi+d r \wedge \omega$ is a smooth representative of $[\alpha]$ and $\beta=\psi+d r \wedge v$ is any other representative. Then there is some form $\eta=\rho+d r \wedge \theta \in L^{2} \Omega^{i-1}\left(C(F), g_{c}\right)$ such that $\beta=\alpha+d_{\max } \eta$, so $\psi=\phi+d_{F} \rho$ for almost all $a$. We know $\phi(a) \in L^{2} \Omega^{i}(F)$ for any $a$, so choose any $a \in(1 / 2,1)$ where $d_{F} \rho$ is defined and such that $\psi(a) \in L^{2} \Omega^{i}(F)$. Since $L^{2}$ cohomology on a compact manifold is the same as absolute cohomology, this means that even if $\rho(a)$ is not in $L^{2}(F)$, there must be some $L^{2}$ form $\tilde{\rho}_{a}$ that is and 
for which $\psi(a)=\phi(a)+d_{F} \tilde{\rho}_{a}$. Thus $[\psi(a)]=[\phi(a)]$, so the map $R$ is independent of the choice of cohomology representatives. To show that it is independent of the choice of $a$, it suffices therefore to consider smooth representatives. For $\alpha$ a representative as before, since $\phi(a) \in L^{2} \Omega^{i}(F)$ for any $a$, we need to show that $[\phi(a)]=[\phi(b)]$ for any $a, b \in(1 / 2,1)$. Since $\alpha$ is smooth, $\int_{a}^{b} \omega(s) d s \in L^{2} \Omega^{i-1}(F)$ and

$$
d \int_{a}^{b} \omega(s) d s=\int_{a}^{b} d_{F} \omega(s) d s=\int_{a}^{b} \phi^{\prime}(s) d s=\phi(b)-\phi(a) .
$$

Thus $[\phi(a)]=[\phi(b)]$, so the map $R$ is well-defined.

To show the map is linear, let $a$ be a value in $(1 / 2,1)$ such that $\alpha(a), \beta(a)$ and $(\alpha+\beta)(a)$ are all in $L^{2} \Omega^{i}(F)$. Then $R([\alpha])+R([\beta])=[\alpha(a)]+[\beta(a)]=[(\alpha+\beta)(a)]=$ $R([\alpha+\beta])$. The map is bounded by Proposition 5 and surjective by Proposition 4 . So we have left to show only that it is injective. So suppose that $R([\alpha])=[0]$ and let $\alpha=\phi+d r \wedge \omega$ be a smooth representative. Then $\phi(a)=d_{F} \eta$ for some $\eta \in$ $L^{2} \Omega^{i-1}(F)$. By Proposition 4, we can consider $\eta$ as a form in $L^{2} \Omega^{i-1}\left(C(F), g_{c}\right)$, and by Proposition $6, \int_{a}^{r} \omega(s) d s \in L^{2} \Omega^{i-1}\left(C(F), g_{c}\right)$. Then

$$
\begin{aligned}
d\left(\eta+\int_{a}^{r} \omega(s) d s\right) & =d_{F} \eta+d r \wedge \omega(r)+\int_{a}^{r} d_{F} \omega(s) d s \\
& =\phi(a)+d r \wedge \omega(r)+\int_{a}^{r} \phi^{\prime}(s) d s=\alpha .
\end{aligned}
$$

Thus $\alpha$ is exact and $[\alpha]=[0]$.

Now consider the case where $i \geq \frac{f}{2}+\frac{1}{2 c}$. Since $c<1$, this implies that $c(f-2 i+2)<1$. We want to show that any class $[\alpha] \in H_{\max }^{i}\left(C(F), g_{c}\right)$ is trivial. First we know again that any such class can be represented by a smooth form. Let $\alpha=\phi+d r \wedge \omega \in$ $L^{2} \Omega^{i}\left(C(F), g_{c}\right)$ be a smooth representative of $[\alpha]$. We need to show that $\alpha=d_{\max } \eta$ for some $\eta \in L^{2} \Omega^{i-1}\left(C(F), g_{c}\right)$. Consider $K_{0}(\alpha)=\int_{0}^{r} \omega(s) d s$. Then

$$
\begin{aligned}
\left\|K_{0}(\alpha)\right\|_{L^{2}\left(C(F), g_{c}\right)}^{2} & =\int_{0}^{1} \int_{F}\left\|\int_{0}^{r} \omega(s) d s\right\|_{F}^{2} r^{c(f-2 i+2)} d \operatorname{vol}_{F} d r . \\
& \leq \int_{0}^{1} \int_{F} r^{c(f-2 i+2)}\left(\int_{0}^{r} s^{-2 j} d s \int_{0}^{r}\left\|s^{j} \omega(s)\right\|_{F}^{2} d s\right) d \operatorname{vol}_{F} d r
\end{aligned}
$$

where the inequality holds for all $j$ by the same steps as in the proof of Proposition 6 . For $2 j<1$, the right-hand side of the equality equals

$$
\frac{1}{-2 j+1} \int_{0}^{1} \int_{F} r^{c(f-2 i+2)} r^{-2 j+1}\left(\int_{0}^{r}\left\|s^{j} \omega(s)\right\|_{F}^{2} d s\right) d \operatorname{vol}_{F} d r .
$$


Let $2 j=c(f-2 i+2)$. Then this becomes

$$
\begin{aligned}
\frac{1}{-c(f-2 i+2)+1} \int_{0}^{1} \int_{F} r \int_{0}^{r} & \|\omega(s)\|_{F}^{2} s^{c(f-2 i+2)} d s d \operatorname{vol}_{F} d r \\
& \leq \frac{1}{-c(f-2 i+2)+1} \int_{0}^{1} r\|\omega\|_{L^{2}\left(C(F), g_{c}\right)}^{2} d r \\
& \leq \frac{1}{-2 c(f-2 i+2)+2}\|\alpha\|_{L^{2}\left(C(F), g_{c}\right)}^{2} .
\end{aligned}
$$

Thus $K_{0}: L^{2} \Omega^{i}\left(C(F), g_{c}\right) \longrightarrow L^{2} \Omega^{i-1}\left(C(F), g_{c}\right)$ is a bounded map, so it extends to $L^{2}$ forms. We want to show that if $\alpha$ is closed, then $d_{\max }\left(K_{0}(\alpha)\right)=\alpha$. This means that we need to show that for any $\beta \in C_{0}^{\infty} \Omega^{i-1}(C(F))$, we have

$$
\left\langle K_{0}(\alpha), \delta \beta\right\rangle_{L^{2}\left(C(F), g_{c}\right)}=\langle\alpha, \beta\rangle_{L^{2}\left(C(F), g_{c}\right)} .
$$

So let $\beta \in C_{0}^{\infty} \Omega^{i-1}(C(F))$. Then for some $\rho>0, \beta$ is supported in $(\rho, 1) \times F$. So equation (17) becomes

$$
\left\langle K_{0}(\alpha), \delta \beta\right\rangle_{L^{2}\left((\rho, 1) \times F, g_{c}\right)}=\langle\alpha, \beta\rangle_{L^{2}\left((\rho, 1) \times F, g_{c}\right)} .
$$

We'd like to use the fundamental theorem of calculus as in the proof for $i<\frac{f}{2}+\frac{1}{2 c}$, but since we don't know that $\alpha$ is continuous at 0 , we can't do this directly. So to get around this we use the following claim:

Claim 1 Define

$$
K_{\epsilon}(\alpha)=\int_{\epsilon}^{r} \omega(s) d s
$$

Then on $(\rho, 1) \times F$ with the restricted $g_{c}$ metric,

$$
K_{\epsilon}(\alpha) \stackrel{L^{2}\left((\rho, 1) \times F, g_{c}\right)}{\longrightarrow} K_{0}(\alpha) .
$$

Proof We have

$$
\left\|K_{\epsilon}(\alpha)-K_{0}(\alpha)\right\|_{L^{2}\left((\rho, 1) \times F, g_{c}\right)}^{2}=\int_{\rho}^{1} \int_{F}\left\|\int_{0}^{\epsilon} \omega(s) d s\right\|_{F}^{2} r^{c(f-2 i+2)} d \operatorname{vol}_{F} d r .
$$

As in the proof of Proposition 6, the right side is at most

$$
\frac{\epsilon^{-c(f-2 i+2)+1}}{-c(f-2 i+2)+1}\left(\int_{\rho}^{1} r^{c(f-2 i+2)} d r\right)\|\omega(s)\|_{L^{2}\left(C(F), g_{c}\right)}^{2} .
$$

Since $c(f-2 i+2)<1$, the first integral in this product converges, and the whole expression tends to 0 as $\epsilon \rightarrow 0$. 
So now we can consider for a closed smooth $\alpha$,

$$
\begin{aligned}
d K_{\epsilon}(\alpha) & =d \int_{\epsilon}^{r} \omega(s) d s=d r \wedge \omega(r)+\int_{\epsilon}^{r} d_{F} \omega(s) d s \\
& =d r \wedge \omega(r)+\int_{\epsilon}^{r} \phi^{\prime}(s) d s=d r \wedge \omega(r)+\phi(r)-\phi(\epsilon)=\alpha-\phi(\epsilon) .
\end{aligned}
$$

By Proposition 7, there is some sequence $\epsilon_{s} \rightarrow 0$ such that

$$
\lim _{\epsilon_{s} \rightarrow 0}\left\|\phi\left(\epsilon_{s}\right)\right\|_{L^{2}\left((\rho, 1) \times F, g_{c}\right)}^{2}=0 .
$$

So our equation (18) can be proved as follows:

$$
\begin{aligned}
\left\langle K_{0}(\alpha), \delta \beta\right\rangle_{L^{2}\left((\rho, 1) \times F, g_{c}\right)} & =\lim _{\epsilon \rightarrow 0}\left\langle K_{\epsilon}(\alpha), \delta \beta\right\rangle_{L^{2}\left((\rho, 1) \times F, g_{c}\right)} \\
& =\lim _{\epsilon \rightarrow 0}\left\langle d K_{\epsilon}(\alpha), \beta\right\rangle_{L^{2}\left((\rho, 1) \times F, g_{c}\right)} \\
& =\langle\alpha, \beta\rangle_{L^{2}\left((\rho, 1) \times F, g_{c}\right)}-\lim _{\epsilon \rightarrow 0}\langle\phi(\epsilon), \beta\rangle_{L^{2}\left((\rho, 1) \times F, g_{c}\right)}
\end{aligned}
$$

Thus the limit

$$
\lim _{\epsilon \rightarrow 0}\langle\phi(\epsilon), \beta\rangle_{L^{2}\left((\rho, 1) \times F, g_{c}\right)}
$$

must exist. This means we can calculate it from any subsequence, such as the subsequence $\epsilon_{s}$ given in Proposition 7. So we get

$$
\begin{aligned}
\lim _{\epsilon \rightarrow 0}\langle\phi(\epsilon), \beta\rangle_{L^{2}\left((\rho, 1) \times F, g_{c}\right)} & =\lim _{\epsilon_{s} \rightarrow 0}\left\langle\phi\left(\epsilon_{S}\right), \beta\right\rangle_{L^{2}\left((\rho, 1) \times F, g_{c}\right)} \\
& \leq \lim _{\epsilon_{s} \rightarrow 0}\left\|\phi\left(\epsilon_{S}\right)\right\|_{L^{2}\left((\rho, 1) \times F, g_{c}\right)}\|\beta\|_{L^{2}\left((\rho, 1) \times F, g_{c}\right)}=0 .
\end{aligned}
$$

So

$$
\left\langle K_{0}(\alpha), \delta \beta\right\rangle_{L^{2}\left((\rho, 1) \times F, g_{c}\right)}=\langle\alpha, \beta\rangle_{L^{2}\left((\rho, 1) \times F, g_{c}\right)},
$$

and we have that $d_{\max } K_{0}(\alpha)=\alpha$ as required.

\section{Proof of signature theorem}

The proof of the signature theorem also comes in three parts. In the first part, we prove that the various signature pairings we will invoke make sense. In the second part, we will prove a version of Novikov additivity, which allows us to calculate the signature near the singular stratum of $X$ separately from the signature of the smooth stratum. Finally, in the third part, we calculate the signature near the singular stratum of $X$ by a close examination of the Leray-Serre spectral sequence of the boundary fibre bundle. 


\subsection{Intersection pairings}

In order to prove Theorem 2, we first need to check that it makes sense to talk about a signature on $\operatorname{Im}\left(I H_{p}^{n / 2}(X) \rightarrow I H_{q}^{n / 2}(X)\right)$, where $p<q$ are dual perversities for $X$.

Lemma 4 Let $p<q$ be dual perversities for intersection cohomology on the compactification $X$ of a manifold with fibre bundle boundary $M$ described in the introduction. There is a well-defined (degenerate) bilinear pairing

$$
I H_{p}^{n / 2}(X) \otimes I H_{p}^{n / 2}(X) \rightarrow \mathbb{R}
$$

given by the map

$$
I H_{p}^{n / 2}(X) \rightarrow I H_{q}^{n / 2}(X)
$$

and the nondegenerate bilinear pairing

$$
I H_{p}^{n / 2}(X) \otimes I H_{q}^{n / 2}(X) \rightarrow \mathbb{R} .
$$

It descends to a well-defined nondegenerate bilinear pairing $B_{X}$

$$
\operatorname{Im}\left(I H_{p}^{n / 2}(X) \rightarrow I H_{q}^{n / 2}(X)\right) \otimes \operatorname{Im}\left(I H_{p}^{n / 2}(X) \rightarrow I H_{q}^{n / 2}(X)\right) \rightarrow \mathbb{R} .
$$

We denote the signature of this pairing by $\sigma_{p}(X)$ and call it the perverse signature for $X$ with perversity $p$.

Proof By Theorem $1, I H_{p}^{*}(X)$ and $I H_{q}^{*}(X)$ can be identified with $H_{\min }^{*}\left(M, g_{c}\right)$ and $H_{\max }^{*}\left(M, g_{c}\right)$, respectively, for some $0 \leq c \leq 1$. To show that the pairing is well-defined and descends to one that is well-defined on $\operatorname{Im}\left(I H_{p}^{n / 2}(X) \rightarrow I H_{q}^{n / 2}(X)\right)$, let $\phi, \psi, \theta \in \mathcal{D}\left(d_{\min }\right)$ be closed and assume that $[\phi]=[\psi] \in H_{\max }^{*}\left(M, g_{c}\right)$. Then $\delta_{\min } * \theta= \pm * d_{\min } \theta=0$ and $\phi=\psi+d_{\max } \eta$ for some $\eta \in \mathcal{D}\left(d_{\max }\right)$. So

$$
\begin{aligned}
\int_{M} \phi \wedge \theta & =\int_{M}\left(\psi+d_{\max } \eta\right) \wedge \theta=\int_{M} \psi \wedge \theta+\int_{M} d_{\max } \eta \wedge \theta \\
& =\int_{M} \psi \wedge \theta \pm \int_{M} d_{\max } \eta \wedge *^{2} \theta=\int_{M} \psi \wedge \theta \pm\left\langle d_{\max } \eta, * \theta\right\rangle \\
& =\int_{M} \psi \wedge \theta \pm\left\langle\eta, \delta_{\min } * \theta\right\rangle=\int_{M} \psi \wedge \theta .
\end{aligned}
$$

Thus the pairing is well-defined and descends to one that is also well-defined. To show it descends to a nondegenerate pairing, we need to show that the subspace

$$
W=\left\{[\phi] \in H_{\min }^{n / 2}\left(M, g_{c}\right) \mid \int_{M} \phi \wedge \psi=0 \text { for all }[\psi] \in H_{\min }^{n / 2}\left(M, g_{c}\right)\right\}
$$


is the same as the kernel of $\iota: H_{\min }^{n / 2}\left(M, g_{c}\right) \rightarrow H_{\max }^{n / 2}\left(M, g_{c}\right)$. Let $[\phi] \in \operatorname{ker}(\iota)$. Then $\phi=d_{\max } \eta$ for some $\eta \in \mathcal{D}\left(d_{\max }\right)$, and for $\psi$ in $\mathcal{D}\left(d_{\min }\right)$ closed, we have

$$
\begin{aligned}
\int_{M} \phi \wedge \psi=\int_{M} d_{\max } \eta \wedge \psi & = \pm \int_{M} d_{\max } \eta \wedge *^{2} \psi \\
& = \pm\left\langle d_{\max } \eta, * \psi\right\rangle= \pm\left\langle\eta, \delta_{\min } * \psi\right\rangle=0 .
\end{aligned}
$$

Thus $[\phi] \in W$. Now suppose that $[\phi]$ is not in $\operatorname{ker}(\iota)$. Then $[\phi]=\iota([\phi]) \neq[0] \in$ $H_{\max }^{n / 2}\left(M, g_{c}\right)$. Thus by Poincaré duality, there is some $[\psi] \in H_{\min }^{n / 2}\left(M, g_{c}\right)$ with $\int_{M} \phi \wedge \psi \neq 0$. Thus $[\phi]$ is not in $W$. So $W$ is exactly $\operatorname{ker}(\iota)$, and we are done.

We also need to define a signature on the cone-bundle neighborhood of the singular stratum of $X$. Let $Z$ be a compact pseudomanifold with boundary, $Y$, that does not intersect the singular part of $Z$. Define $I H_{p}^{i}(Z)$ to be the cohomology of the cochain complex $I C^{*}(Z)$, which has $p$-perversity intersection cohomology conditions near the singular set of $Z$ and with absolute cohomology conditions at the boundary, $Y$. That is to say, define it as the cohomology of global sections for a complex of fine sheaves for which the local calculation near the boundary is the standard one, and the local calculation near the singular stratum is as in the definition of standard intersection cohomology of perversity $p$. Define $I H_{p, 0}^{i}(Z, Y)$ to be the cohomology of the cochain complex $I C^{*}(Z) / I C^{*}(Y)$. The standard arguments from regular cohomology show that this satisfies a relative long exact sequence (see Goresky and MacPherson [13, p 209] or King [17]). Here we are still considering the generalized definition of intersection cohomology near the singular stratum of $X$ as in equation (11), but for simplicity of notation, we will suppress the $B$.

Define a (degenerate) pairing on

$$
I H_{p, 0}^{i}(Z, Y) \otimes I H_{p, 0}^{n-i}(Z, Y)
$$

via the map

$$
I H_{p, 0}^{i}(Z, Y) \longrightarrow I H_{q, 0}^{i}(Z, Y) \longrightarrow I H_{q}^{i}(Z)
$$

and the nondegenerate pairing, $\widetilde{B}_{Z Y}$

$$
I H_{p, 0}^{i}(Z, Y) \otimes I H_{q}^{n-i}(Z) \longrightarrow \mathbb{R} .
$$

When $i=n / 2$, this descends to a well-defined, nondegenerate pairing $B_{Z Y}$ :

$$
\operatorname{Im}\left(I H_{p, 0}^{n / 2}(Z, Y) \rightarrow I H_{q}^{n / 2}(Z)\right) \otimes \operatorname{Im}\left(I H_{p, 0}^{n / 2}(Z, Y) \rightarrow I H_{q}^{n / 2}(Z)\right) \rightarrow \mathbb{R} .
$$

Call its signature $\widehat{\sigma}_{p}(Z)$. In the special case that $Z$ has no singular stratum other than its boundary, $Y$, we suppress the $p$, since the signature will be the same for any perversity, and will simply be the signature of the intersection pairing on $\operatorname{Im}\left(H^{n / 2}(Z, \partial Z) \rightarrow\right.$ 
$\left.H^{n / 2}(Z)\right)$. The proof that this pairing is well-defined and nondegenerate is similar to the proof of Lemma 4 .

\subsection{Novikov additivity}

Next we need a theorem that allows us to study the signature of a neighborhood of the boundary separately from that of the interior of $M$. We use a version of Novikov additivity for this.

Theorem 3 If $X$ is a compact pseudomanifold with a single compact smooth singular stratum and if $Y \subset X$ is a compact codimension 1 submanifold such that $X=Z \cup_{Y} Z^{\prime}$ where $Z \subset \subset X^{\text {reg }}$, then

$$
\sigma_{p}(X)=\hat{\sigma}(Z)+\widehat{\sigma}_{p}\left(Z^{\prime}\right)
$$

Proof The proof is a modification of the original Novikov additivity theorem in Atiyah and Singer [2]. Assume that $X$ is $n$ dimensional, and let

and

$$
\begin{aligned}
\hat{H}^{n / 2}(Z) & =\operatorname{Im}\left(H^{n / 2}(Z, Y) \rightarrow H^{n / 2}(Z)\right) \\
\widehat{I H}_{p}^{n / 2}\left(Z^{\prime}\right) & =\operatorname{Im}\left(I H_{p, 0}^{n / 2}\left(Z^{\prime}, Y\right) \rightarrow I H_{q}^{n / 2}\left(Z^{\prime}\right)\right)
\end{aligned}
$$

Using the exact relative cohomology sequences for $I H_{p}^{*}(X)$ and $I H_{q}^{*}(X)$, we have:

$$
\begin{aligned}
& \cdots \longrightarrow I H_{p, 0}^{n / 2}(X, Z) \stackrel{\alpha_{p}^{\prime}}{\longrightarrow} I H_{p}^{n / 2}(X) \stackrel{\beta_{p}}{\longrightarrow} I H_{p}^{n / 2}(Z) \longrightarrow \cdots \\
& \cdots \longrightarrow I H_{q, 0}^{n / 2}(X, Z) \stackrel{\alpha_{q}^{\prime}}{\longrightarrow} I H_{q}^{n / 2}(X) \stackrel{\beta_{q}}{\longrightarrow} I H_{q}^{n / 2}(Z) \longrightarrow \cdots \\
& \cdots \lessdot \quad I H_{q}^{n / 2}\left(Z^{\prime}\right) \ll \stackrel{\beta_{q}^{\prime}}{\longleftarrow} I H_{q}^{n / 2}(X) \longleftarrow \frac{\alpha_{q}}{\longleftarrow} I H_{q, 0}^{n / 2}\left(X, Z^{\prime}\right) \longleftarrow \cdots \\
& \cdots \longleftarrow I H_{p}^{n / 2}\left(Z^{\prime}\right) \stackrel{\beta_{p}^{\prime}}{\longleftarrow} I H_{p}^{n / 2}(X) \stackrel{\alpha_{p}}{\longleftarrow} I H_{p, 0}^{n / 2}\left(X, Z^{\prime}\right) \longleftarrow \cdots
\end{aligned}
$$


Because $Z$ is smooth, these sequences are the same as:

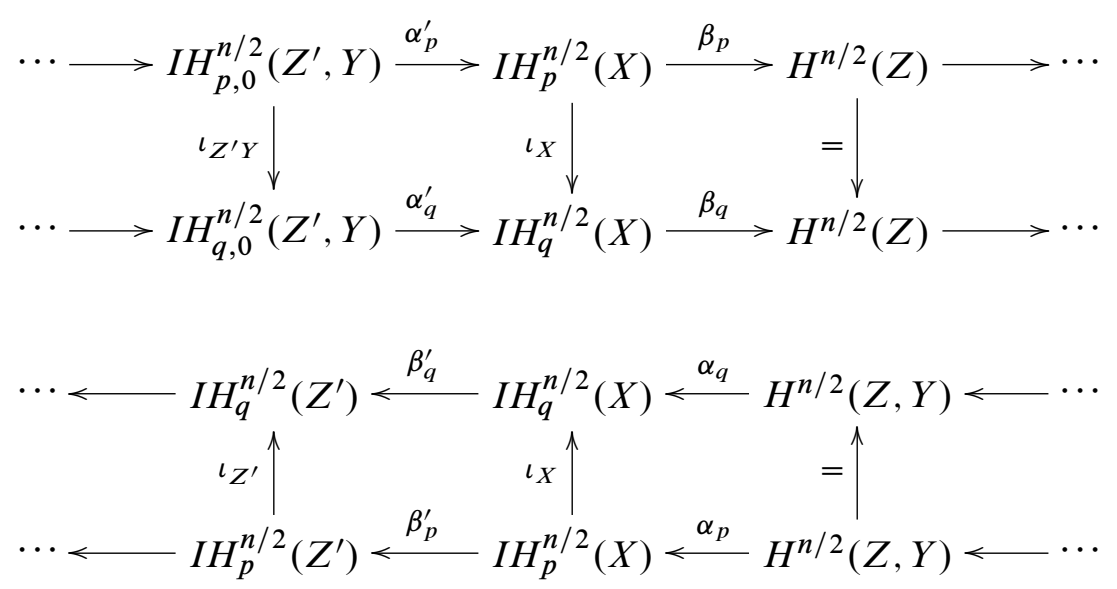

The maps ${ }^{\prime} Z^{\prime} Y, l^{\prime} Z^{\prime}$ and $\iota_{X}$ are induced from the natural inclusion maps on the complexes of forms, and this entire diagram commutes. Further, the maps are natural with respect to the intersection pairings.

Define

$$
\begin{aligned}
A^{\prime} & =\operatorname{Im}\left(\iota_{X} \circ \alpha_{p}^{\prime}\right) \subset \operatorname{ker}\left(\beta_{q}\right) \\
A & =\operatorname{Im}\left(\alpha_{q}\right)=\operatorname{ker}\left(\beta_{q}^{\prime}\right)=\operatorname{Im}\left(\iota_{X} \circ \alpha_{p}\right) .
\end{aligned}
$$

Proposition 8 The spaces $A$ and $A^{\prime}$ are mutual annihilators in $\operatorname{Im}\left(I H_{p}^{n / 2}(X) \rightarrow\right.$ $\left.I H_{q}^{n / 2}(X)\right)$ under the intersection pairing $B_{X}$.

Proof Suppose that $\iota_{X} \circ \alpha_{p}^{\prime}[a] \in A^{\prime}$ and $\alpha_{q}[b] \in A$. Then

$$
B_{X}\left(\iota_{X} \circ \alpha_{p}^{\prime}[a], \alpha_{q}[b]\right)=\widetilde{B}_{Z^{\prime} Y}\left([a], \beta_{q}^{\prime} \circ \alpha_{q}[b]\right)=0 .
$$

So $A$ and $A^{\prime}$ are contained in each other's annihilators.

Now suppose that $[e] \in \operatorname{Im}\left(I H_{p}^{n / 2}(X) \rightarrow I H_{q}^{n / 2}(X)\right)$ annihilates $A^{\prime}$ under $B_{X}$. Then for every $\left[c^{\prime}\right] \in I H_{p, 0}^{n / 2}\left(Z^{\prime}, Y\right)$,

$$
0=B_{X}\left(\iota_{X} \circ \alpha_{p}^{\prime}\left[c^{\prime}\right],[e]\right)=\widetilde{B}_{Z^{\prime} Y}\left(\left[c^{\prime}\right], \beta_{q}^{\prime}[e]\right) .
$$

Since the pairing $\widetilde{B}_{Z^{\prime} Y}$ is nondegenerate, this can happen only if $\beta_{q}^{\prime}[e]=0$. But then by exactness of the $I H_{q}$ sequence we have $[e] \in \operatorname{Im}\left(\alpha_{q}\right)=A$. So $A$ is the annihilator of $A^{\prime}$ under $B_{Z}$.

Finally, suppose that $\iota_{X}\left[e^{\prime}\right] \in \operatorname{Im}\left(I H_{p}^{n / 2}(X) \rightarrow I H_{q}^{n / 2}(X)\right)$ annihilates $A$ under $B_{X}$. Then for all $[c] \in H^{n / 2}(Z, Y)$,

$$
0=B_{X}\left(\alpha_{q}[c], \iota_{X}\left[e^{\prime}\right]\right)=\widetilde{B}_{Z Y}\left([c], \beta_{q} \circ \iota_{X}\left[e^{\prime}\right]\right)=\widetilde{B}_{Z Y}\left([c], \beta_{p}\left[e^{\prime}\right]\right)
$$


This implies that $\beta_{p}\left[e^{\prime}\right]=0$, so by exactness of the $I H_{p}$ sequence, $\left[e^{\prime}\right] \in \operatorname{Im}\left(\alpha_{p}^{\prime}\right)$. So ${ }^{\iota_{X}}\left[e^{\prime}\right] \in A^{\prime}$, and we get that $A^{\prime}$ is also the annihilator of $A$.

Since the pairing $B_{X}$ is nondegenerate, we get as in the original Novikov additivity proof that

We also have

$$
\left(A \cap A^{\prime}\right)^{*} \cong \frac{\operatorname{Im}\left(I H_{p}^{n / 2}(X) \rightarrow I H_{q}^{n / 2}(X)\right)}{A+A^{\prime}}
$$

$$
\frac{A+A^{\prime}}{A \cap A^{\prime}}=\frac{A}{A \cap A^{\prime}} \oplus \frac{A^{\prime}}{A \cap A^{\prime}} \cong \frac{\operatorname{Im}\left(\alpha_{q}\right)}{\operatorname{Im}\left(\alpha_{q}\right) \cap \operatorname{Im}\left(\iota_{X} \circ \alpha_{p}^{\prime}\right)} \oplus \frac{\operatorname{Im}\left(\iota_{X} \circ \alpha_{p}^{\prime}\right)}{\operatorname{Im}\left(\alpha_{q}\right) \cap \operatorname{Im}\left(\iota_{X} \circ \alpha_{p}^{\prime}\right)} .
$$

From the exact sequences, we know that

$$
\begin{aligned}
& \frac{\operatorname{Im}\left(\iota_{X} \circ \alpha_{p}^{\prime}\right)}{\operatorname{Im}\left(\alpha_{q}\right) \cap \operatorname{Im}\left(\iota_{X} \circ \alpha_{p}^{\prime}\right)} \cong \frac{\operatorname{Im}\left(\iota_{X} \circ \alpha_{p}^{\prime}\right)}{\operatorname{ker}\left(\beta_{q}^{\prime}\right) \cap \operatorname{Im}\left(\iota_{X} \circ \alpha_{p}^{\prime}\right)} \cong \operatorname{Im}\left(\beta_{q}^{\prime} \circ \iota_{X} \circ \alpha_{p}^{\prime}\right) \cong \hat{I H}_{p}^{n / 2}\left(Z^{\prime}\right) \\
& \text { and also that } \\
& \frac{\operatorname{Im}\left(\alpha_{q}\right)}{\operatorname{Im}\left(\alpha_{q}\right) \cap \operatorname{Im}\left(\iota_{X} \circ \alpha_{p}^{\prime}\right)} \cong \frac{\operatorname{Im}\left(\iota_{X} \circ \alpha_{p}\right)}{\operatorname{Im}\left(\iota_{X} \circ \alpha_{p}\right) \cap \iota_{X}\left(\operatorname{ker}\left(\beta_{p}\right)\right)} \\
& \cong \frac{\operatorname{Im}\left(\iota_{X} \circ \alpha_{p}\right)}{\operatorname{Im}\left(\iota_{X} \circ \alpha_{p}\right) \cap \operatorname{Im}\left(\iota_{X}\right) \cap \operatorname{ker}\left(\beta_{q}\right)} .
\end{aligned}
$$

Since $\operatorname{Im}\left(\iota_{X} \circ \alpha_{p}\right) \subset \operatorname{Im}\left(\iota_{X}\right)$, we can eliminate the $\operatorname{Im}\left(\iota_{X}\right)$ term in the denominator to get

$$
\frac{\operatorname{Im}\left(\iota_{X} \circ \alpha_{p}\right)}{\operatorname{Im}\left(\iota_{X} \circ \alpha_{p}\right) \cap \operatorname{ker}\left(\beta_{q}\right)} \cong \frac{\operatorname{Im}\left(\alpha_{q}\right)}{\operatorname{Im}\left(\alpha_{q}\right) \cap \operatorname{ker}\left(\beta_{q}\right)} \cong \hat{H}^{n / 2}(Z)
$$

So altogether, we have

$$
\frac{A+A^{\prime}}{A \cap A^{\prime}} \cong \hat{H}^{n / 2}(Z) \oplus \widehat{I H}_{p}^{n / 2}\left(Z^{\prime}\right) .
$$

By splitting the maps

$$
A \cap A^{\prime} \rightarrow A+A^{\prime} \rightarrow \operatorname{Im}\left(I H_{p}^{n / 2}(X) \rightarrow I H_{q}^{n / 2}(X)\right)
$$

we get that

$$
\operatorname{Im}\left(I H_{p}^{n / 2}(X) \rightarrow I H_{q}^{n / 2}(X)\right) \cong \hat{H}^{n / 2}(Z) \oplus \hat{I H}_{p}^{n / 2}\left(Z^{\prime}\right) \oplus\left(\left(A \cap A^{\prime}\right) \oplus\left(A \cap A^{\prime}\right)^{*}\right) .
$$

As in the proof of the original Novikov additivity, by choosing a good splitting, we can arrange for the form $B_{X}$ to be given with respect to this splitting by the block matrix

$$
\left(\begin{array}{lll}
B_{Z Y} & 0 & 0 \\
0 & B_{Z^{\prime} Y} & 0 \\
0 & 0 & C
\end{array}\right)
$$


where $C$ is the natural form on $\left(\left(A \cap A^{\prime}\right) \oplus\left(A \cap A^{\prime}\right)^{*}\right)$. The signature of such a form is always 0 [2, p 209], so we obtain

$$
\sigma_{p}(X)=\widehat{\sigma}_{p}(Z)+\widehat{\sigma}_{p}\left(Z^{\prime}\right)
$$

as we wanted.

\subsection{Signature of the end}

Now return to our original situation, where $\bar{M}$ is a compact $n$ dimensional manifold with boundary $Y \stackrel{\phi}{\rightarrow} B$ with fibre $F$, and $X$ is the pseudomanifold formed by coning off the fibres of $Y$. As before, let $f$ be the dimension of $F$ and $b$ be the dimension of $B$ so that $f+b+1=n$. Decompose $X$ as $M \cup_{Y} C_{\phi} Y$, where

$$
C_{\phi} Y=Y \times[0,1] /\left\{(y, 0) \sim\left(y^{\prime}, 0\right) \text { if } \phi(y)=\phi\left(y^{\prime}\right)\right\}
$$

is the pseudomanifold with boundary formed by coning off the fibres of $Y$ on one end of a finite cylinder. Assume that $p=\underline{\mathfrak{m}}-k$ and $q=\overline{\mathfrak{m}}+k$. By Theorem 3, to prove Theorem 2, it suffices to prove the following:

Lemma 5 The signature on $C_{\phi} Y$ is

$$
\hat{\sigma}_{p}\left(C_{\phi} Y\right)=-\sum_{r=2+2 k}^{\infty} \tau_{r}
$$

Once we have this, reversing orientation to glue, we get

$$
\sigma_{p}(X)=\hat{\sigma}(M)+\widehat{\sigma}_{p}\left(C_{\phi} Y\right)=\operatorname{sgn} \operatorname{Im}\left(H^{n / 2}(M, \partial M) \rightarrow H^{n / 2}(M)\right)+\sum_{r=2+2 k}^{\infty} \tau_{r}
$$

as required.

The proof of Lemma 5 generalizes the techniques of Cheeger and Dai [7].

Proof The first step is to identify the image $\operatorname{Im}\left(I H_{p, 0}^{*}\left(C_{\phi} Y, Y\right) \rightarrow I H_{q, 0}^{*}\left(C_{\phi} Y, Y\right)\right)$ and $I H_{q}^{*}\left(C_{\phi} Y\right)$ and in terms of the Leray-Serre spectral sequence for the fibre bundle on $Y$. 
Proposition 9 With respect to the $E_{\infty}^{i, j}\left(H^{*}(Y)\right)$ terms of the Leray-Serre spectral sequence for $Y$ and its differentials $d_{s}^{i, j}: E_{s}^{i, j}\left(H^{*}(Y)\right) \rightarrow E_{s}^{i+s, j-s+1}\left(H^{*}(Y)\right)$, we get the isomorphisms:

$$
\begin{aligned}
& I H_{q}^{n / 2}\left(C_{\phi} Y\right) \\
& \cong \bigoplus_{\substack{\max (0, b-n / 2) \leq a \\
\leq \min (b,(b-1) / 2+k)}} E_{\infty}^{b-a,(n / 2)-b+a}\left(H^{*}(Y)\right) \oplus \sum_{\substack{\max (0, b-n / 2) \leq l \\
\leq b / 2-k-1}} \operatorname{Im}\left(-d_{b-a-l}^{l, n / 2-1-l}\right) \\
& \operatorname{Im}\left(I H_{p, 0}^{n / 2}\left(C_{\phi} Y, Y\right) \rightarrow I H_{q, 0}^{n / 2}\left(C_{\phi} Y, Y\right)\right) \\
& \cong \bigoplus_{\substack{\max (0, b-n / 2) \leq a \\
\leq \min (b, b / 2-1-k)}} E_{\infty}^{a, n / 2-a-1}\left(H^{*}(Y)\right) \oplus \sum_{\substack{0 \leq l \\
\leq(b-1) / 2-k}} \operatorname{Im}\left(-d_{b-a-l}^{a, n / 2-a-1}\right)^{*}
\end{aligned}
$$

Proof Recall that by Theorem $1, I H_{q}^{*}\left(C_{\phi} Y\right) \cong H_{\max }^{*}\left(C_{\phi} Y, g_{c}\right)$ and $I H_{p}^{*}\left(C_{\phi} Y\right) \cong$ $H_{\mathrm{min}}^{*}\left(C_{\phi} Y, g_{c}\right)$ for some metric $g_{c}, 0 \leq c \leq 1$, where $H_{\min }^{*}\left(C_{\phi} Y, g_{c}\right)$ means cohomology that is minimal with respect to the metric $g_{c}$ near the singular stratum of $C_{\phi} Y$ and absolute near the boundary $Y \times\{1\}$. The same proofs as in the case of regular cohomology show that relative intersection cohomology may in this case be calculated either from the cochain complex of forms in $L^{2} \Omega^{*}\left(C_{\phi} Y, g\right)$ that vanish in a neighborhood of the boundary, $Y \times\{1\}$, or from the cochain complex $\Omega^{i-1}(Y) \oplus L^{2} \Omega^{i}(M, g)$ with the relative differential $d_{\text {rel }}(\theta, \beta)=\left(-d_{Y} \theta-\beta(1), d_{M} \beta\right)$. We will use this second formulation to prove the proposition. The short exact sequence

$$
0 \rightarrow \Omega^{*-1}(Y) \stackrel{\gamma}{\rightarrow} \Omega^{*-1}(Y) \oplus \Omega_{\min }^{*}\left(C_{\phi} Y, g_{c}\right) \stackrel{\beta}{\rightarrow} \Omega_{\min }^{*}\left(C_{\phi} Y, g_{c}\right) \rightarrow 0
$$

induces a long exact sequence on cohomologies. Similarly, for perversity $q$ cohomologies, we get a long exact sequence arising from the short exact sequence on forms:

$$
0 \rightarrow \Omega^{*-1}(Y) \stackrel{\gamma}{\rightarrow} \Omega^{*-1}(Y) \oplus \Omega_{\max }^{*}\left(C_{\phi} Y\right) \stackrel{\beta}{\rightarrow} \Omega_{\max }^{*}\left(C_{\phi} Y\right) \rightarrow 0
$$

Using the metric $g_{c}$, each of these spaces of forms can be filtered by (conic) fibre and base bi-degree as in Section 2.2. Consider the cohomologies with maximal boundary condition near the singularity first. For any base degree, $i$, we get the following 
commutative diagram relating maximal, ie $q$-perversity, complexes:

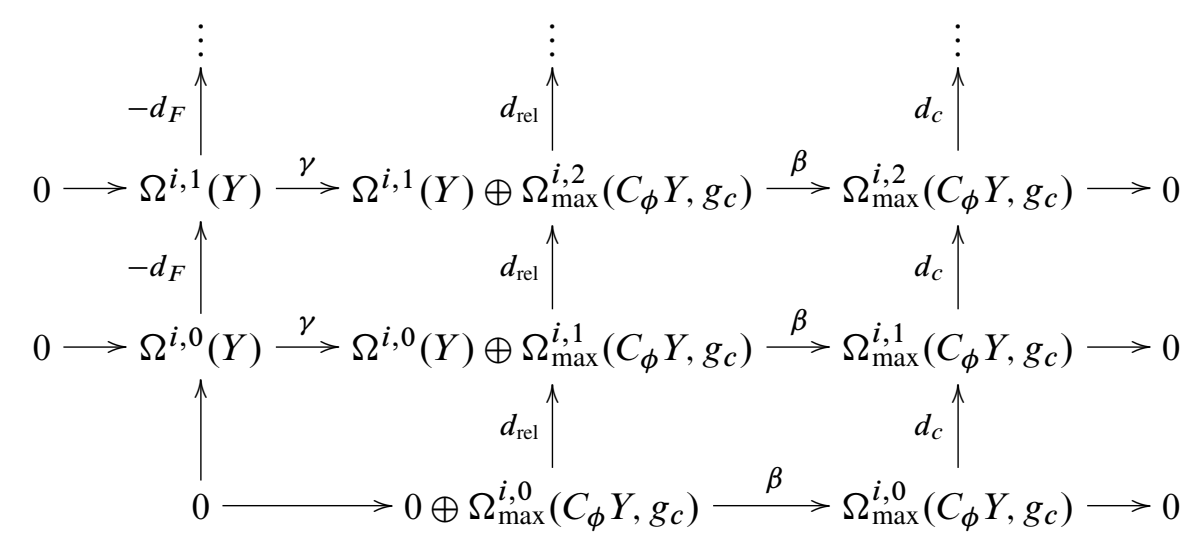

Here the first column gives $E_{0}^{i, *-1}\left(H^{*}(Y)\right)$ terms, the second column gives

$E_{0}^{i, *}\left(I H_{q, 0}^{*}\left(C_{\phi} Y, Y\right)\right)$ terms and the third column gives $E_{0}^{i, *}\left(I H_{q}^{*}\left(C_{\phi} Y\right)\right)$ terms. Taking vertical differentials gives $E_{1}$ for each of these spectral sequences. Thus the $E_{1}$ terms of the spectral sequences for each base degree $i$ fit into the long exact sequence:

$$
\begin{aligned}
\cdots \rightarrow E_{1}^{i, j-1}\left(I H_{q}^{*}\left(C_{\phi} Y\right)\right) \rightarrow E_{1}^{i, j-1} & \left(H^{*}(Y)\right) \rightarrow E_{1}^{i, j}\left(I H_{q, 0}^{*}\left(C_{\phi} Y, Y\right)\right) \\
& \rightarrow E_{1}^{i, j}\left(I H_{q}^{*}\left(C_{\phi} Y\right)\right) \rightarrow E_{1}^{i, j}\left(H^{*}(Y)\right) \rightarrow \cdots .
\end{aligned}
$$

By the Poincaré lemma for the maximal complex, Lemma 3, we get that for $j \leq \frac{f-1}{2}+k$ if $f$ is odd and $j \leq \frac{f}{2}+k$ if $f$ is even, this is

$$
\begin{aligned}
\rightarrow \Omega^{i}\left(B, H^{j-1}(F)\right) \rightarrow \Omega^{i}\left(B, H^{j-1}(F)\right) & \rightarrow E_{1}^{i, j}\left(I H_{q, 0}^{*}\left(C_{\phi} Y, Y\right)\right) \rightarrow \\
& \rightarrow \Omega^{i}\left(B, H^{j}(F)\right) \rightarrow \Omega^{i}\left(B, H^{j}(F)\right) \rightarrow
\end{aligned}
$$

So for these values of $j$, we find $E_{1}^{i, j}\left(I H_{q, 0}^{*}\left(C_{\phi} Y, Y\right)\right)=0$. For $j \geq \frac{f-1}{2}+k+2$ if $f$ is odd and $j \geq \frac{f}{2}+k+2$ if $f$ is even, the long exact sequence is

$$
\cdots \rightarrow 0 \rightarrow \Omega^{i}\left(B, H^{j-1}(F)\right) \rightarrow E_{1}^{i, j}\left(I H_{q, 0}^{*}\left(C_{\phi} Y, Y\right)\right) \rightarrow 0 \rightarrow \Omega^{i}\left(B, H^{j}(F)\right) \rightarrow \cdots .
$$

So for $j \geq \frac{f-1}{2}+k+2$ if $f$ is odd and $j \leq \frac{f}{2}+k+2$ if $f$ is even, we find $E_{1}^{i, j}\left(I H_{q, 0}^{*}\left(C_{\phi} Y, Y\right)\right) \cong \Omega^{i}\left(B, H^{j-1}(F)\right)$. Thus for the boundary degree $j=\frac{f-1}{2}+$ $k+1$ if $f$ is odd, we have (dropping the last term and adding one previous term in 
the long exact sequence):

$$
\begin{aligned}
\rightarrow 0 \rightarrow \Omega^{i}\left(B, H^{(f-1) / 2+k}(F)\right) \rightarrow \Omega^{i} & \left(B, H^{(f-1) / 2+k}(F)\right) \rightarrow \\
\rightarrow & E_{1}^{i,(f-1) / 2+k+1}\left(I H_{q, 0}^{*}\left(C_{\phi} Y, Y\right)\right) \rightarrow 0 \rightarrow
\end{aligned}
$$

So if $f$ is odd,

$$
E_{1}^{i,(f-1) / 2+k+1}\left(I H_{q, 0}^{*}\left(C_{\phi} Y, Y\right)\right)=0 .
$$

If $f$ is even we get similarly that

$$
E_{1}^{i, f / 2+k+1}\left(I H_{q, 0}^{*}\left(C_{\phi} Y, Y\right)\right)=0 .
$$

The differential $d_{1}$ is horizontal, so it does not see the truncations. Thus the $E_{2}$ pages of the relative and absolute $q$-perversity spectral sequences are for $f$ odd:

Relative $q$ sequence $\left(E_{2}^{i, j}\left(I H_{q, 0}^{*}\left(C_{\phi} Y, Y\right)\right)\right)$ :

$$
\begin{array}{lllll}
j=f+1 & H^{0}\left(B, H^{f}(F)\right) & H^{1}\left(B, H^{f}(F)\right) & H^{1}\left(B, H^{f}(F)\right) & \cdots \\
\vdots & \vdots & \vdots & \vdots & \cdots \\
j=\frac{f+3}{2}+k & H^{0}\left(B, H^{\frac{f+1}{2}+k}(F)\right) & H^{1}\left(B, H^{\frac{f+1}{2}+k}(F)\right) & H^{2}\left(B, H^{\frac{f+1}{2}+k}(F)\right) & \cdots \\
j=\frac{f+1}{2}+k & 0 & 0 & 0 & \cdots \\
\vdots & \vdots & \vdots & \vdots & \cdots \\
j=0 & 0 & 0 & 0 & \cdots \\
& i=0 & i=1 & i=2 & \cdots
\end{array}
$$

Absolute $q$ sequence $\left(E_{2}^{i, j}\left(I H_{q}^{*}\left(C_{\phi} Y\right)\right)\right)$ :

$\begin{array}{lllll}\vdots & \vdots & \vdots & \vdots & \\ j=\frac{f+1}{2}+k & 0 & 0 & 0 & \cdots \\ j=\frac{f-1}{2}+k & H^{0}\left(B, H^{\frac{f-1}{2}+k}(F)\right) & H^{1}\left(B, H^{\frac{f-1}{2}+k}(F)\right) & H^{2}\left(B, H^{\frac{f-1}{2}+k}(F)\right) & \cdots \\ \vdots & \vdots & \vdots & \vdots & \cdots \\ j=0 & H^{0}\left(B, H^{0}(F)\right) & H^{1}\left(B, H^{0}(F)\right) & H^{2}\left(B, H^{0}(F)\right) & \cdots \\ & i=0 & i=1 & i=2 & \cdots\end{array}$


And using an analogous argument for the minimal complex, we get that the $E_{2}$ term of the relative $p$-perversity spectral sequence $\left(E_{2}^{i, j}\left(I H_{p, 0}^{*}\left(C_{\phi} Y, Y\right)\right)\right)$ is:

$\begin{array}{lllll}j=f+1 & H^{0}\left(B, H^{f}(F)\right) & H^{1}\left(B, H^{f}(F)\right) & H^{1}\left(B, H^{f}(F)\right) & \cdots \\ \vdots & \vdots & \vdots & \vdots & \cdots \\ j=\frac{f+3}{2}-k & H^{0}\left(B, H^{\frac{f+1}{2}-k}(F)\right) & H^{1}\left(B, H^{\frac{f+1}{2}-k}(F)\right) & H^{2}\left(B, H^{\frac{f+1}{2}-k}(F)\right) & \cdots \\ j=\frac{f+1}{2}-k & 0 & 0 & 0 & \cdots \\ \vdots & \vdots & \vdots & \vdots & \cdots \\ j=0 & 0 & 0 & 0 & \cdots \\ & i=0 & i=1 & i=2 & \cdots\end{array}$

In the case that $f$ is even, these diagrams are similar, but for the relative $q$ spectral sequence, one has $E_{2}^{i, j}\left(I H_{q, 0}^{*}\left(C_{\phi} Y, Y\right)\right)=0$ for $j<f / 2+2+k$ and otherwise $E_{2}^{i, j}\left(I H_{q, 0}^{*}\left(C_{\phi} Y, Y\right)\right)=H^{i}\left(B, H^{j-1}(F)\right)$, as in the odd case. For the absolute $q$ spectral sequence, we have $E_{2}^{i, j}\left(I H_{q, 0}^{*}\left(C_{\phi} Y\right)\right)=0$ for $j>f / 2+k$ and otherwise $E_{2}^{i, j}\left(I H_{q, 0}^{*}\left(C_{\phi} Y\right)\right)=H^{i}\left(B, H^{j}(F)\right)$. For the relative $p$ spectral sequence, for $j<$ $f / 2+1-k$ one has $E_{2}^{i, j}\left(I H_{p, 0}^{*}\left(C_{\phi} Y, Y\right)\right)=0$ and otherwise $E_{2}^{i, j}\left(I H_{p, 0}^{*}\left(C_{\phi} Y, Y\right)\right)=$ $H^{i}\left(B, H^{j-1}(F)\right)$. The terms of $E_{2}^{i, j}\left(I H_{p, 0}^{*}\left(C_{\phi} Y, Y\right)\right)$ are all equal to 0 for $j>f+1$ and for $i>b$. The terms of $E_{2}^{i, j}\left(I H_{q}^{*}\left(C_{\phi} Y\right)\right)$ are all equal to 0 for $j>f$ and for $i>b$.

These are all truncated (and in the relative case, shifted) copies of the Leray-Serre spectral sequence for the fibre bundle on $Y$, although with the negatives of the differentials. So, the higher pages of these spectral sequences will be the same as the higher pages of the spectral sequence for $Y$, but with terms added because of the truncation. We need to understand what the extra terms in all of these various related spectral sequences look like. On page $r$ of one of the absolute intersection cohomology spectral sequences, we will pick a term of the form $\operatorname{Im}\left(d_{r}^{i, j}\right)$ that come from cases where there is no longer any image to quotient by because of the truncation above. By definition, elements in $E_{r+1}^{*, *}$ have representatives in $E_{r}^{*, *}$ which are in the kernel of $d_{r}$, so this extra term is simply carried through on page $r+1$ and each subsequent page. Thus all of the extra terms in $E_{\infty}\left(I H_{q}^{*}\left(C_{\phi} Y\right)\right)$ will have the form of $\operatorname{Im}\left(d_{s}^{i, j}\right)$ and will come from cases where there is no longer any image to quotient by because of the truncation above. Similarly, in the cases of $E_{\infty}\left(I H_{q, 0}^{*}\left(C_{\phi} Y, Y\right)\right)$ and $E_{\infty}\left(I H_{p, 0}^{*}\left(C_{\phi} Y, Y\right)\right)$, the extra terms will have the form $\operatorname{Im}\left(d_{r}^{i, j}\right)^{*}$ and will come from the cases where, because of the truncation below, the kernel of the differential in the truncated sequence contains the image of the adjoint of the differential in the spectral sequence for $Y$. 
It is easiest to understand this in an example. Take the case when the base dimension $b=6$, the fibre dimension $f=5$, and $k=1$, so the perversities are $p=\mathfrak{m}-1$ and $q=\overline{\mathfrak{m}}+1$. Recall that $d_{r}^{i, j}: E_{r}^{i, j} \longrightarrow E_{r}^{i+r, j-r+1}$. For compactness of notation, in these diagrams, we will denote $\operatorname{Im}\left(-d_{r}^{i, j}\right)$ simply by $\left(d_{r}^{i, j}\right)$ and $\operatorname{Im}\left(-d_{r}^{i, j}\right)^{*}$ simply by $\left(d_{r}^{i, j}\right)^{*}$. In Proposition 10, we will see that the negatives before the differentials do not in fact make a difference in the final calculations, but we will preserve them except in the diagrams below. Also, from this point forward we will use the notation $E_{\infty}^{i, j}$ and $d_{r}^{i, j}$ to refer to the underlying spectral sequence for $Y$ rather than for the various relative and absolute intersection cohomologies it spawns. We get the following diagrams.

Example $\left(E_{\infty}^{i, j}\left(I H_{\mathfrak{m}+1,0}^{*}\left(C_{\phi} Y, Y\right)\right)\right.$ : Relative cohomology spectral sequence $)$

$$
\begin{aligned}
& j=6 \quad E_{\infty}^{0,5}+\left(d_{3}^{0,5}\right)^{*} E_{\infty}^{1,5}+\left(d_{3}^{1,5}\right)^{*} E_{\infty}^{2,5}+\left(d_{3}^{2,5}\right)^{*} E_{\infty}^{3,5}+\left(d_{3}^{3,5}\right)^{*} E_{\infty}^{4,5} \quad E_{\infty}^{5,5} E_{\infty}^{6,5} \\
& +\cdots+\left(d_{6}^{0,5}\right)^{*}+\cdots+\left(d_{5}^{1,5}\right)^{*}+\left(d_{4}^{2,5}\right)^{*} \\
& j=5 \quad E_{\infty}^{0,4}+\left(d_{2}^{0,4}\right)^{*} \quad E_{\infty}^{1,4}+\left(d_{2}^{1,4}\right)^{*} \quad E_{\infty}^{2,4}+\left(d_{2}^{2,4}\right)^{*} \quad E_{\infty}^{3,4}+\left(d_{2}^{3,4}\right)^{*} \quad E_{\infty}^{4,4} \quad E_{\infty}^{5,4} \quad E_{\infty}^{6,4} \\
& +\cdots+\left(d_{5}^{0,4}\right)^{*}+\cdots+\left(d_{5}^{1,4}\right)^{*}+\cdots+\left(d_{4}^{2,4}\right)^{*}+\left(d_{3}^{3,4}\right)^{*}+\left(d_{2}^{4,4}\right)^{*} \\
& \begin{array}{llllllll}
j=4 & 0 & 0 & 0 & 0 & 0 & 0 & 0 \\
\vdots & \vdots & \vdots & \vdots & \vdots & \vdots & \vdots & \vdots \\
j=0 & 0 & 0 & 0 & 0 & 0 & 0 & 0 \\
& i=0 & i=1 & i=2 & i=3 & i=4 & i=5 & i=6
\end{array}
\end{aligned}
$$

Example $\left(E_{\infty}^{i, j}\left(I H_{\overline{\mathfrak{m}}+1}^{*}\left(C_{\phi} Y\right)\right):\right.$ Absolute cohomology spectral sequence $)$

$$
\begin{aligned}
& \begin{array}{llllllll}
j=5 & 0 & 0 & 0 & 0 & 0 & 0 & 0
\end{array} \\
& j=4 \begin{array}{lllllll}
j & 0 & 0 & 0 & 0 & 0 & 0
\end{array} \\
& j=3 \quad E_{\infty}^{0,3} \quad E_{\infty}^{1,3} \quad E_{\infty}^{2,3}+\left(d_{2}^{0,4}\right) \quad E_{\infty}^{3,3}+\left(d_{2}^{1,4}\right) \quad E_{\infty}^{4,3}+\left(d_{2}^{2,4}\right) E_{\infty}^{5,3}+\left(d_{2}^{3,4}\right) E_{\infty}^{6,3}+\left(d_{2}^{4,4}\right) \\
& +\left(d_{3}^{0,5}\right)+\left(d_{3}^{1,5}\right)+\left(d_{3}^{2,5}\right)+\left(d_{3}^{3,5}\right) \\
& j=2 \quad E_{\infty}^{0,2} \quad E_{\infty}^{1,2} \quad E_{\infty}^{2,2} \quad E_{\infty}^{3,2}+\left(d_{3}^{0,4}\right) E_{\infty}^{4,2}+\left(d_{3}^{1,4}\right) E_{\infty}^{5,2}+\left(d_{3}^{2,4}\right) E_{\infty}^{6,2}+\left(d_{3}^{3,4}\right) \\
& +\left(d_{4}^{0,5}\right) \quad+\left(d_{4}^{1,5}\right) \quad+\left(d_{4}^{2,5}\right) \\
& j=1 \quad E_{\infty}^{0,1} \quad E_{\infty}^{1,1} \quad E_{\infty}^{2,1} \quad E_{\infty}^{3,1} \quad E_{\infty}^{4,1}+\left(d_{4}^{0,4}\right) E_{\infty}^{5,1}+\left(d_{4}^{1,4}\right) E_{\infty}^{6,1}+\left(d_{4}^{2,4}\right) \\
& +\left(d_{5}^{0,5}\right) \quad+\left(d_{5}^{1,5}\right) \\
& j=0 \quad E_{\infty}^{0,0} \quad E_{\infty}^{1,0} \quad E_{\infty}^{2,0} \quad E_{\infty}^{3,0} \quad E_{\infty}^{4,0} \quad E_{\infty}^{5,0}+\left(d_{5}^{0,4}\right) E_{\infty}^{6,0}+\left(d_{5}^{1,4}\right) \\
& i=0 \quad i=1 \quad i=2 \quad i=3 \quad i=4 \quad i=5 \quad i=6 \\
& +\left(d_{6}^{0,5}\right)
\end{aligned}
$$


Example $\left(E_{\infty}^{i, j}\left(I H_{\mathfrak{m}-1,0}^{*}\left(C_{\phi} Y, Y\right)\right):\right.$ Relative cohomology spectral sequence $)$

$$
\begin{aligned}
& j=6 E_{\infty}^{0,5}+\left(d_{5}^{0,5}\right)^{*} E_{\infty}^{1,5}+\left(d_{5}^{1,5}\right)^{*} E_{\infty}^{2,5} \quad E_{\infty}^{3,5} \quad E_{\infty}^{4,5} \quad E_{\infty}^{5,5} E_{\infty}^{6,5} \\
& +\left(d_{6}^{0,5}\right)^{*} \\
& j=5 E_{\infty}^{0,4}+\left(d_{4}^{0,4}\right)^{*} E_{\infty}^{1,4}+\left(d_{4}^{1,4}\right)^{*} E_{\infty}^{2,4}+\left(d_{4}^{2,4}\right)^{*} E_{\infty}^{3,4} \quad E_{\infty}^{4,4} \quad E_{\infty}^{5,4} \quad E_{\infty}^{6,4} \\
& +\left(d_{5}^{0,4}\right)^{*} \quad+\left(d_{5}^{1,4}\right)^{*} \\
& j=4 \quad E_{\infty}^{0,3}+\left(d_{3}^{0,3}\right)^{*} E_{\infty}^{1,3}+\left(d_{3}^{1,3}\right)^{*} E_{\infty}^{2,3}+\left(d_{3}^{2,3}\right)^{*} E_{\infty}^{3,3}+\left(d_{3}^{3,3}\right)^{*} E_{\infty}^{4,3} \quad E_{\infty}^{5,3} E_{\infty}^{6,3} \\
& +\left(d_{4}^{0,3}\right)^{*} \quad+\left(d_{4}^{1,3}\right)^{*} \quad+\left(d_{4}^{2,3}\right)^{*} \\
& j=3 \quad E_{\infty}^{0,2}+\left(d_{2}^{0,2}\right)^{*} \quad E_{\infty}^{1,2}+\left(d_{2}^{1,2}\right)^{*} \quad E_{\infty}^{2,2}+\left(d_{2}^{2,2}\right)^{*} \quad E_{\infty}^{3,2}+\left(d_{2}^{3,2}\right)^{*} E_{\infty}^{4,2} \quad E_{\infty}^{5,2} \quad E_{\infty}^{6,2} \\
& +\left(d_{3}^{0,2}\right)^{*} \quad+\left(d_{3}^{1,2}\right)^{*} \quad+\left(d_{3}^{2,2}\right)^{*} \quad+\left(d_{3}^{3,2}\right)^{*} \quad+\left(d_{2}^{4,2}\right)^{*} \\
& \begin{array}{llllllll}
j=2 & 0 & 0 & 0 & 0 & 0 & 0 & 0 \\
j=1 & 0 & 0 & 0 & 0 & 0 & 0 & 0 \\
j=0 & 0 & 0 & 0 & 0 & 0 & 0 & 0 \\
& i=0 & i=1 & i=2 & i=3 & i=4 & i=5 \quad i=6
\end{array}
\end{aligned}
$$

In this example, we get for instance:

$$
\begin{aligned}
& E_{\infty}^{2,5}\left(I H_{\mathfrak{m}+1,0}^{*}\left(C_{\phi} Y, Y\right)\right)=E_{\infty}^{2,4}+\operatorname{Im}\left(-d_{2}^{2,4}\right)^{*}+\operatorname{Im}\left(-d_{3}^{2,4}\right)^{*}+\operatorname{Im}\left(-d_{4}^{2,4}\right)^{*}, \\
& E_{\infty}^{3,3}\left(I H_{\mathfrak{m}+1}^{*}\left(C_{\phi} Y\right)\right)=E_{\infty}^{3,3}+\operatorname{Im}\left(-d_{2}^{1,4}\right)+\operatorname{Im}\left(-d_{3}^{0,5}\right), \\
& E_{\infty}^{2,5}\left(I H_{\underline{m}-1,0}^{*}\left(C_{\phi} Y, Y\right)\right)=E_{\infty}^{2,4}+\operatorname{Im}\left(-d_{4}^{2,4}\right)^{*} \text {. }
\end{aligned}
$$

In general we get that:

$$
\begin{aligned}
& E_{\infty}^{i, j}\left(I H_{q, 0}^{*}\left(C_{\phi} Y, Y\right)\right) \cong \begin{cases}0 & j<(f+3) / 2+k \\
E_{\infty}^{i, j-1}+\sum_{\substack{j-f / 2-k \\
\leq s \leq b-i}} \operatorname{Im}\left(-d_{s}^{i, j-1}\right)^{*} & j \geq(f+3) / 2+k \\
& i+j<(f+1) / 2 \\
E_{\infty}^{i, j-1} & +b+k \\
& \text { otherwise, }\end{cases} \\
& E_{\infty}^{i, j}\left(I H_{q}^{*}\left(C_{\phi} Y\right)\right) \cong \begin{cases}0 & j>f / 2+k \\
E_{\infty}^{i, j}+\sum_{(f+3) / 2-j+k} \operatorname{Im}\left(-d_{s}^{i-s, j+s-1}\right) & j \leq f / 2+k, \\
E_{\infty}^{i, j} & i+j>(f+1) / 2+k\end{cases}
\end{aligned}
$$


and

$$
E_{\infty}^{i, j}\left(I H_{p, 0}^{*}\left(C_{\phi} Y, Y\right)\right) \cong \begin{cases}0 & j<f / 2+1-k \\ E_{\infty}^{i, j-1}+\sum_{\substack{j-f / 2+k \\ \leq s \leq b-i}} \operatorname{Im}\left(-d_{s}^{i, j-1}\right)^{*} & j \geq f / 2+1-k, \\ E_{\infty}^{i, j-1} & i+j<f / 2+b-k\end{cases}
$$

When we map $I H_{p, 0}^{*}\left(C_{\phi} Y, Y\right)$ to $I H_{q, 0}^{*}\left(C_{\phi} Y, Y\right)$ we simply truncate the bottom $2 k$ rows of $E_{\infty}^{i, j}\left(I H_{p}^{*}\left(C_{\phi} Y, Y\right)\right)$ if $f$ is odd, and the bottom $2 k+1$ rows if $f$ is even. Summing over $i+j=n / 2=(b+f+1) / 2$, we get:

$$
\begin{aligned}
& I H_{q}^{n / 2}\left(C_{\phi} Y\right) \cong \begin{cases}\bigoplus_{\substack{i+j=n / 2 \\
j \leq f / 2+k}} E_{\infty}^{i, j}\left(H^{*}(Y)\right) & b / 2-k>0 \\
\quad \oplus \sum_{s \geq(f+3) / 2+k-j}^{i} \operatorname{Im}\left(-d_{s}^{i-s, j+s-1}\right) & \\
\bigoplus_{\substack{i+j=n / 2 \\
j \leq f / 2+k}}^{i, j}\left(H^{*}(Y)\right) & b / 2-k \leq 0\end{cases} \\
& \operatorname{Im}\left(I H_{p, 0}^{n / 2}\left(C_{\phi} Y, Y\right) \rightarrow I H_{q, 0}^{n / 2}\left(C_{\phi} Y, Y\right)\right) \\
& \cong\left\{\begin{array}{cc}
\bigoplus_{\substack{i+j=n / 2 \\
j \geq(f+3) / 2+k}} E_{\infty}^{i, j-1}\left(H^{*}(Y)\right) & b / 2-k>0 \\
\quad \oplus \sum_{s \geq j-f / 2+k}^{b-i} \operatorname{Im}\left(-d_{s}^{i, j-1}\right)^{*} & \\
\bigoplus_{\substack{i+j=n / 2 \\
j \geq(f+3) / 2+k}}^{i, j-1}\left(H^{*}(Y)\right) & b / 2-k \leq 0 .
\end{array}\right.
\end{aligned}
$$

The result of the proposition then follows by reindexing.

Now we want to understand the signature pairing on

$$
\operatorname{Im}\left(I H_{p, 0}^{n / 2}\left(C_{\phi} Y, Y\right) \rightarrow I H_{q, 0}^{n / 2}\left(C_{\phi} Y\right)\right)
$$

in terms of this decomposition. To do this, first return to our example where $f=5$, $b=6$ and $k=1$. We have

$$
\begin{aligned}
I H_{\mathfrak{m}+1}^{6}\left(C_{\phi} Y\right) \cong E_{\infty}^{6,0}\left(I H_{q}^{*}\left(C_{\phi} Y\right)\right) & +E_{\infty}^{5,1}\left(I H_{q}^{*}\left(C_{\phi} Y\right)\right) \\
& +E_{\infty}^{4,2}\left(I H_{q}^{*}\left(C_{\phi} Y\right)\right)+E_{\infty}^{3,3}\left(I H_{q}^{*}\left(C_{\phi} Y\right)\right)
\end{aligned}
$$

and $\operatorname{Im}\left(I H_{\underline{\mathfrak{m}}-1,0}^{6}\left(C_{\phi} Y, Y\right) \rightarrow I H_{\mathfrak{m}+1,0}^{6}\left(C_{\phi} Y, Y\right)\right)$

$$
\begin{aligned}
\cong \operatorname{Im}\left(E_{\infty}^{0,6}\left(I H_{p}^{*}\left(C_{\phi} Y, Y\right)\right) \rightarrow E_{\infty}^{0,6}\left(I H_{q}^{*}\left(C_{\phi} Y, Y\right)\right)\right) \\
\quad+\operatorname{Im}\left(E_{\infty}^{1,5}\left(I H_{p}^{*}\left(C_{\phi} Y, Y\right)\right) \rightarrow E_{\infty}^{1,5}\left(I H_{q}^{*}\left(C_{\phi} Y, Y\right)\right)\right),
\end{aligned}
$$

where these sums are the outside sums in the proposition. Only forms with complementary bi-degrees for the cone bundle $C_{\phi} Y$ can have nontrivial pairing. By Serre's filtration 
on Leray spectral sequences (see Chern, Hirzebruch and Serre [8]), we can always represent a class in $E_{r}^{i, j}\left(I H_{q}^{*}\left(C_{\phi} Y\right)\right)$ or $E_{r}^{i, j}\left(I H_{p, 0}^{*}\left(C_{\phi} Y, Y\right)\right)$ by a form $\theta$ that is a sum of forms of bidegree $(i+h, j-h)$ for $h \geq 0$ and for which $d \theta$ is a sum of forms whose base degree is at least $i+r$. A pair of terms whose $i$ indices add to more than the dimension of the base will have trivial intersection. Therefore in our example, we can represent the intersection form on $I H_{\mathfrak{m}+1}^{6}\left(C_{\phi} Y\right) \otimes \operatorname{Im}\left(I H_{\mathfrak{m}-1,0}^{6}\left(C_{\phi} Y, Y\right) \rightarrow I H_{\mathfrak{m}+1,0}^{6}\left(C_{\phi} Y, Y\right)\right)$ by a 2 by 4 block lower-triangular matrix

$$
\left(\begin{array}{llll}
A^{0} & 0 & 0 & 0 \\
* & A^{1} & 0 & 0
\end{array}\right)
$$

where $A^{0}$ is the pairing between

$$
\operatorname{Im}\left(E_{\infty}^{0,6}\left(I H_{p, 0}^{*}\left(C_{\phi} Y, Y\right)\right) \rightarrow E_{\infty}^{0,6}\left(I H_{q, 0}^{*}\left(C_{\phi} Y, Y\right)\right)\right)
$$

and $E_{\infty}^{6,0}\left(I H_{q}^{*}\left(C_{\phi} Y\right)\right)$ and $A^{1}$ is the pairing between

$$
\operatorname{Im}\left(E_{\infty}^{1,5}\left(I H_{p, 0}^{*}\left(C_{\phi} Y, Y\right)\right) \rightarrow E_{\infty}^{1,5}\left(I H_{q, 0}^{*}\left(C_{\phi} Y, Y\right)\right)\right)
$$

and $E_{\infty}^{5,1}\left(I H_{q}^{*}\left(C_{\phi} Y\right)\right)$.

In general, by this argument, $A$ will be a block lower triangular matrix with blocks $A^{a}, \max (0, b-n / 2) \leq a \leq b / 2-k-1$, along the diagonal that represent the pairings between

$$
\operatorname{Im}\left(E_{\infty}^{a, n / 2-a}\left(I H_{\underline{\mathfrak{m}}-k, 0}^{*}\left(C_{\phi} Y, Y\right)\right) \rightarrow E_{\infty}^{a, n / 2-a}\left(I H_{\underline{\mathfrak{m}}+k, 0}^{*}\left(C_{\phi} Y, Y\right)\right)\right)
$$

and

$$
E_{\infty}^{b-a, n / 2-b+a}\left(I H_{\overline{\mathfrak{m}}+k}^{*}\left(C_{\phi} Y\right)\right) .
$$

Note that this same argument allows us to simplify $\tau_{r}$. By definition, $\tau_{r}$ corresponds to the signature of the pairing $E_{r}^{(b+f-1) / 2} \otimes E_{r}^{(b+f-1) / 2} \longrightarrow \mathbb{R}$. Decomposing by bidegree, we can consider it as the signature of the block matrix whose blocks come from the decomposition of $E_{r}^{(b+f-1) / 2}$. By the argument above, this must be a block lower triangular matrix with blocks $T_{r}^{j}$ along the diagonal corresponding to the pairings $E_{r}^{p,(b+f-1) / 2-p} \otimes E_{r}^{b-p-r,(f-b-1) / 2+p+r} \longrightarrow \mathbb{R}$.

Now we have to understand the pieces $A^{a}$. First consider $A^{0}$ in our example. We can further decompose $A^{0}$ into a 3 by 3 block matrix corresponding to the decompositions:

$$
\begin{gathered}
E_{\infty}^{6,0}\left(I H_{q}^{*}\left(C_{\phi} Y\right)\right) \cong E_{\infty}^{6,0}+\operatorname{Im}\left(-d_{6}^{0,5}\right)+\operatorname{Im}\left(-d_{5}^{1,4}\right) \\
\operatorname{Im}\left(E_{\infty}^{0,6}\left(I H_{p, 0}^{*}\left(C_{\phi} Y, Y\right)\right) \rightarrow E_{\infty}^{0,6}\left(I H_{q, 0}^{*}\left(C_{\phi} Y, Y\right)\right)\right) \\
\cong E_{\infty}^{0,5}+\operatorname{Im}\left(-d_{6}^{0,5}\right)^{*}+\operatorname{Im}\left(-d_{5}^{0,5}\right)^{*}
\end{gathered}
$$


where recall that as before, by $E_{\infty}^{i, j}$ and $-d_{r}^{i, j}$ we refer to the spectral sequence for $Y$. In general, $A^{a}$ will decompose into a block matrix whose block rows and block columns correspond to the inside sums in Proposition 9:

$$
E_{\infty}^{b-a,(n / 2)-b+a}\left(H^{*}(Y)\right) \oplus \sum_{\substack{\max (0, b-n / 2) \leq l \\ \leq b / 2-k-1}} \operatorname{Im}\left(-d_{b-a-l}^{l, n / 2-1-l}\right)
$$

and

$$
E_{\infty}^{a, n / 2-a-1}\left(H^{*}(Y)\right) \oplus \sum_{0 \leq l \leq(b-1) / 2-k} \operatorname{Im}\left(-d_{b-a-l}^{a, n / 2-a-1}\right)^{*} .
$$

In the case that $f$ is odd, $A^{a}$ is a $b / 2-k$ by $b / 2-k-\max (0, b-n / 2)$ block matrix. In the case that $f$ is even, it is a $(b+1) / 2-k$ by $(b-1) / 2-k-\max (0, b-n / 2)$ block matrix. In either case, the diagonal will consist of one block matrix representing the pairing of $E_{\infty}$ terms, and further matrices $A_{l}^{a}$ representing the pairings between

$$
\operatorname{Im}\left(-d_{b-a-l}^{a, n / 2-a-1}\right)^{*} \text { and } \operatorname{Im}\left(-d_{b-a-l}^{l, n / 2-1-l}\right),
$$

for $\max (0, b-n / 2) \leq l \leq b / 2-k-1$. Note that $l$ has exactly the same range as $a$.

Modifying slightly the argument from [7] we have the following:

Proposition 10 In terms of the spectral sequence, the map

$$
I H_{q, 0}^{*}\left(C_{\phi} Y, Y\right) \rightarrow I H_{q}^{*}\left(C_{\phi} Y\right)
$$

is zero on the terms of the form $E_{\infty}^{i, j}\left(H^{*}(Y)\right)$ and is given on the other factors by applying the appropriate $-d_{r}$ 's.

Proof First consider a form $[\theta]$ in the $\operatorname{Im}\left(-d_{r}^{i, j-1}\right)^{*}$ piece of $I H_{q, 0}^{*}\left(C_{\phi} Y, Y\right)$. In terms of the construction of the relative cohomology spectral sequence, this is really a representative $[\theta, 0]$ on page $r$. This is not necessarily a class in the limiting cohomology, so we would like to find an equivalent class that is. By definition of the spectral sequence, we can alter our representative $[\theta, 0]$ by anything of the form $d_{\text {rel }}\left(\psi_{1}, \psi_{2}\right)+\left(\xi_{1}, \xi_{2}\right)$, where $\psi_{1}$ and $\psi_{2}$ have base degree $\geq i+1-r$, the components of $d_{\text {rel }}\left(\psi_{1}, \psi_{2}\right)$ have base degree $\geq i, \xi_{1}$ and $\xi_{2}$ have base degree $\geq i+1$, and $d_{\text {rel }}\left(\xi_{1}, \xi_{2}\right)$ has base degree $\geq i+s$.

A piece of the form $\operatorname{Im}\left(-d_{r}^{i, j-1}\right)^{*}$ appears in $I H_{q, 0}^{*}\left(C_{\phi} Y, Y\right)$ exactly when its elements $[\theta]$ are mapped by $-d_{r}$ to $E_{r}^{i+r, j-r}$, where $j-r$ is below the level of truncation for the spectral sequence on $Y$. That is, if $j-r \leq(f-1) / 2+k$ when $f$ is odd and if $j-r \leq f / 2+k$ when $f$ is even. So we can choose $\theta$ so that $-d_{Y} \theta$ will have fibre degree $\leq(f-1) / 2+k$ if $f$ is odd and $\leq f / 2+k$ if $f$ is even. From Theorem 1 , 
we know that in terms of maximal cohomology on $\left(C_{\phi} Y, g_{c}\right), k=\llbracket 1 / 2 c \rrbracket$ if $f$ is even and $k=[[1 / 2+1 / 2 c]]$ if $f$ is odd, where $\llbracket x \rrbracket$ is the greatest integer strictly less than $x$. So in either case, the fibre degree of $-d_{Y} \theta$ is less than $f / 2+1 / 2 c$. By Proposition 4, this guarantees that $-d_{Y} \theta$ extends to an $L^{2}$ form on $C_{\phi} Y$ with base degree $\geq i+r$. Also, $d_{c}\left(-d_{Y} \theta\right)=0$, so we may alter our representative $[\theta, 0]$ in $I H_{q, 0}^{*}\left(C_{\phi} Y, Y\right)$ by $\left[0,-d_{Y} \theta\right]$.

We thus obtain a new representative $[\theta,-d \theta]$ that is closed under $d_{\text {rel }}$ and so represents a class in $I H_{q}^{*}\left(C_{\phi} Y, Y\right)$. Under the map to absolute cohomology, this goes to $[-d \theta] \in$ $I H_{q}^{*}\left(C_{\phi} Y\right)$. The map from $\Omega^{*}\left(C_{\phi} Y, g_{c}\right)$ to $\Omega^{*}(Y)$ that induces the identification of $I H_{q}^{*}\left(C_{\phi} Y\right)$ with spectral sequence terms for $Y$ is given by restriction. Since $\theta$ is the lift to $C_{\phi} Y$ of a form on $Y$, we have $-d \theta=-d_{Y} \theta$, which in terms of spectral sequence classes is just $-d_{S}[\theta]$.

Now suppose that $[\theta] \in E_{\infty}^{i, j-1}\left(H^{*}(Y)\right)$. Then $-d_{Y} \theta=0$, so by the same argument as above, we get that $[\theta]$ goes to $[0]$ under the map $I H_{q, 0}^{*}\left(C_{\phi} Y, Y\right) \rightarrow I H_{q}^{*}\left(C_{\phi} Y\right)$.

We know that the entire intersection pairing descends to a nondegenerate pairing on

$$
\operatorname{Im}\left(I H_{p, 0}^{n / 2}\left(C_{\phi} Y, Y\right) \rightarrow I H_{q}^{n / 2}\left(C_{\phi} Y\right)\right)
$$

which by the proposition contains no terms of the form $E_{\infty}^{i, j}$. Thus such terms must pair trivially with everything. So the first row and the first column of block matrices in $A$ are trivial. The rest of the terms pair according to the following proposition. In this proposition only, we change the notation for the page of the spectral sequence from $r$ to $s$ since here, as in Section 3, $r$ will denote the radial variable in $C_{\phi} Y$.

Proposition 11 If $[\theta] \in \operatorname{Im}\left(-d_{s}^{i, j}\right)^{*}$ and $[\alpha] \in \operatorname{Im}\left(-d_{s}^{i, j}\right)$, then their intersection pairing is given by

$$
\langle[\theta],[\alpha]\rangle=-\int_{Y} \theta \wedge \alpha
$$

Before proving this, we note that this proposition together with the previous one implies that the pairing between classes $[\theta]$ and $[\alpha]=d_{s}[\beta]$ in $\operatorname{Im}\left(-d_{s}^{(b+f-1) / 2}\right)^{*} \otimes$ $\operatorname{Im}\left(d_{s}^{(b+f-1) / 2}\right)$ is

$$
\left\langle[\theta], d_{s}[\beta]\right\rangle=\left(\theta \wedge d_{s} \beta, \xi_{s}\right),
$$

which corresponds exactly to the pairing of classes in $E_{s}^{(b+f-1) / 2} \otimes E_{s}^{(b+f-1) / 2}$, whose signature defines $\tau_{s}$. 
Proof From the exact sequences in (19), under the identifications above, an element $[\theta] \in E_{\infty}^{i, j}+\sum \operatorname{Im}\left(-d_{s}^{i, j}\right)^{*}$ lifts to $[\theta,-d \theta] \in I H_{p, 0}^{i+j+1}\left(C_{\phi} Y, Y\right)$, which is constant in the $r$-direction on $C_{\phi} Y$, and an element $[\alpha] \in E_{\infty}^{i, j}+\sum \operatorname{Im}\left(-d_{s}^{i, j}\right)$ lifts to $[\alpha] \in$ $I H_{q}^{i+j}\left(C_{\phi} Y\right)$. Now we want to determine the intersection pairing for lifts in the middle degree, $[\theta,-d \theta] \in I H_{p, 0}^{n / 2}\left(C_{\phi} Y, Y\right)$ and $[\alpha] \in I H_{q}^{n / 2}\left(C_{\phi} Y\right)$. For forms on $C_{\phi} Y$, the pairing is given by integration, so we need to use the map of complexes from $\Omega^{*-1}(Y) \oplus \Omega^{*}\left(C_{\phi} Y\right)$ to $\Omega^{*}\left(C_{\phi} Y, Y\right)$ that induces the isomorphism on cohomology to turn our class $[\theta,-d \theta]$ into a class on $C_{\phi} Y$. Call this map $\mu$.

We will derive the pairing first for general $[\gamma, \beta]$ and $[\alpha]$, not necessarily arising from the spectral sequence identification. The pair $[\gamma, \beta]$ goes under $\mu^{*}$ to the class $\left[(1-\eta) \beta+\eta^{\prime} d r \wedge \gamma\right]$, where as before, $\eta$ is a smooth cutoff function equal to 1 near $Y \times\{1\}$ and equal to 0 near the singular stratum of $C_{\phi} Y$. So the pairing of $[\gamma, \beta]$ and $[\alpha]$ is given by

$$
\int_{C_{\phi} Y}\left((1-\eta) \beta+\eta^{\prime} d r \wedge \gamma\right) \wedge \alpha
$$

Integrating this by parts, it becomes

$$
\begin{aligned}
& \int_{C_{\phi} Y}(1-\eta) \beta \wedge \alpha-\int_{C_{\phi} Y} \eta d(\gamma \wedge \alpha)-\int_{Y} \gamma \wedge \alpha(1) . \\
&=\int_{C_{\phi} Y}(1-\eta) \beta \wedge \alpha-\int_{C_{\phi} Y} \eta d \gamma \wedge \alpha \pm \int_{C_{\phi} Y} \eta \gamma \wedge d \alpha-\int_{Y} \gamma \wedge \alpha(1) .
\end{aligned}
$$

We know that $(\gamma, \beta)$ and $(\alpha)$ are closed under their respective differentials, and $\gamma$ is constant in the $r$ direction, so this equals

$$
\int_{C_{\phi} Y}(1-\eta) \beta \wedge \alpha-\int_{C_{\phi} Y} \eta(-\beta) \wedge \alpha-\int_{Y} \gamma \wedge \alpha(1)=\int_{C_{\phi} Y} \beta \wedge \alpha-\int_{Y} \gamma \wedge \alpha(1) \text {. }
$$

Now returning to the lifts in question, $[\theta] \in E_{\infty}^{i, j}+\sum \operatorname{Im}\left(-d_{s}^{i, j}\right) *$ and $[\alpha] \in E_{\infty}^{i, j}+$ $\sum \operatorname{Im}\left(-d_{s}^{i, j}\right)$, we know that $\alpha$ is constant in $r$, so we get that the pairing between $[\theta,-d \theta]$ and $[\alpha]$ is given by

$$
-\int_{C_{\phi} Y} d \theta \wedge \alpha-\int_{Y} \theta \wedge \alpha
$$

Since neither $d \theta$ nor $\alpha$ contains a $d r$, the first integral vanishes, and we are left with the pairing on our original classes given by the proposition.

We claim that the submatrices $A^{a}$ are also lower block triangular. To see this, consider a pairing above the diagonal. This corresponds to $\langle[\theta],[\alpha]\rangle$, where $[\theta]$ is in on a later page of the spectral sequence for $Y$ than $[\alpha]$ is. For instance, in our example we have the 
pairing of $[\theta] \in \operatorname{Im}\left(-d_{6}^{0,5}\right)^{*}$ and $[\alpha] \in \operatorname{Im}\left(-d_{5}^{1,4}\right)$. More generally the superdiagonal corresponds to pairings

$$
[\theta] \in \operatorname{Im}\left(-d_{b-a-(l-t)}^{a, n / 2-a-1}\right)^{*} \quad \text { and } \quad[\alpha] \in \operatorname{Im}\left(-d_{b-a-l}^{l, n / 2-1-l}\right) .
$$

Then we can choose $\theta$ to be a sum of forms with base degree greater than or equal to $a$ and such that $d \theta$ has base degree greater than or equal to $i+r=b-l+t$. Similarly, we can choose $\alpha$ to have base degree greater than or equal to $l+(b-a-l)=b-a$ and $[\alpha]=d_{b-a-l}[\eta]$ for a form $\eta$ of base degree greater than or equal to $l$. Then $\alpha=d \eta+$ terms of higher base degree. Only the lowest base degree parts of these forms will pair nondegenerately on $Y$, so

$$
\langle[\theta],[\alpha]\rangle=-\int_{Y} \theta \wedge \alpha=-\int_{Y} \theta \wedge d \eta=\int_{Y} d \theta \wedge \eta=0,
$$

since $d \theta$ has base degree $\geq b-l+t$ and $\eta$ has base degree $\geq l$. Thus the block matrix for $A^{a}$ is also of lower triangular form:

$$
A^{a}=\left(\begin{array}{lllll}
0 & 0 & 0 & \cdots & 0 \\
0 & A_{0}^{a} & 0 & \cdots & 0 \\
0 & * & A_{1}^{a} & \cdots & 0 \\
0 & * & * & \ddots & 0 \\
0 & * & * & * & A_{[b / 2-k-1]}^{a}
\end{array}\right),
$$

where $A_{l}^{a}$ is the intersection matrix for $\operatorname{Im}\left(d_{b-a-l}^{a, n / 2-1-a}\right)^{*} \otimes \operatorname{Im}\left(d_{b-a-l}^{l, n / 2-1-l}\right)$.

Now we turn our attention back to the matrix whose signature is $\tau_{r}$, recalling an argument originally from [10], and also appearing in [7]. Recall that this must be a block lower triangular matrix with blocks $T_{r}^{p}$ along the diagonal corresponding to the pairings

$$
E_{r}^{p,(b+f-1) / 2-p} \otimes E_{r}^{b-p-r,(f-b-1) / 2+p+r} \longrightarrow \mathbb{R} .
$$

There is a natural inner product on $E_{2} \cong \oplus H^{p}\left(B, \mathcal{H}^{q}(F)\right)$. With respect to this inner product, we can decompose $E_{2}=E_{3} \oplus \operatorname{Im}\left(d_{2}\right) \oplus \operatorname{Im}\left(d_{2}\right)^{*}$. This in turn induces an inner product on $E_{3}$. Continuing inductively we get inner products on all $E_{r}$. Denote these by $(\cdot, \cdot)_{r}$. Now define the operator $\star_{r}$ by

$$
\left\langle\phi \cdot \psi, \xi_{r}\right\rangle=\left(\phi, \star_{r} \psi\right)_{r} .
$$

In terms of this operator, we find that the signature of $\tau_{r}$ is equal to the signature of the self-adjoint operator $\star_{r} d_{r}$. Note that

$$
\star_{r} d_{r}: E_{r}^{p, q} \longrightarrow E_{r}^{b-p-r, f-q+r-1} .
$$


When we restrict to the spaces appearing in the matrices along the block diagonal, we get that $p=b-p-r$ exactly when

$$
q=(b+f-1) / 2-p=f-((b+f-1) / 2-p)+r-1=f-q+r-1 .
$$

Thus $\star_{r} d_{r}$ descends to an operator on $E_{r}^{(b-r) / 2,(f+r-1) / 2}$. On the complement of this space,

$$
\sum_{p>(b-r) / 2} E_{r}^{p,(b+f-1) / 2-p} \oplus \sum_{p<(b-r) / 2} E_{r}^{p,(b+f-1) / 2-p},
$$

the operator $\star_{r} d_{r}$ takes the form:

$$
\left(\begin{array}{cc}
0 & S \\
S^{*} & 0
\end{array}\right)
$$

This implies that the eigenvalues on this piece cancel, and $\tau_{r}$ is the sum of eigenvalues of $\star_{r} d_{r}$ on $E_{r}^{(b-r) / 2,(f+r-1) / 2}$. Back in terms of the pairing $\left\langle\cdot \wedge d_{r} \cdot, \xi_{r}\right\rangle$, this means that only the pairings of forms on

$$
\operatorname{Im}\left(d_{r}^{\frac{b-r}{2}, \frac{f+r-1}{2}}\right) \otimes \operatorname{Im}\left(d_{r}^{\frac{b-r}{2}, \frac{f+r-1}{2}}\right)
$$

contribute to the signature, $\tau_{r}$. Note in particular that when $f$ and $r$ are both odd or both even, $\tau_{r}=0$.

Finally, we can see by this same argument that only the terms $A_{a}^{a}$ where $\max (0, b-$ $n / 2) \leq a \leq b / 2-k-1$ contribute to the signature of the end. This is because $\star_{b-a-l} d_{b-a-l}$ takes

$$
E_{b-a-l}^{a, n / 2-1-a} \text { to } E_{b-a-l}^{l, n / 2-1-l},
$$

so the contributions coming from the matrices $A_{l}^{a}$ correspond to the contribution from a submatrix of matrix (20) of the form:

$$
\left(\begin{array}{cc}
0 & \tilde{S} \\
\tilde{S}^{*} & 0
\end{array}\right)
$$

The signature of $A_{a}^{a}$ equals $\tau_{b-2 a}$ by the above, so we obtain finally that

$$
\hat{\sigma}_{p}\left(C_{\phi} Y\right)=-\sum_{r=2+2 k}^{\infty} \tau_{r}
$$

and we are done. 


\section{References}

[1] M F Atiyah, V K Patodi, I M Singer, Spectral asymmetry and Riemannian geometry. I, Math. Proc. Cambridge Philos. Soc. 77 (1975) 43-69 MR0397797

[2] MF Atiyah, I M Singer, The index of elliptic operators. III, Ann. of Math. (2) 87 (1968) 546-604 MR0236952

[3] J-M Bismut, J Cheeger, $\eta$-invariants and their adiabatic limits, J. Amer. Math. Soc. 2 (1989) 33-70 MR966608

[4] J Brüning, M Lesch, Hilbert complexes, J. Funct. Anal. 108 (1992) 88-132 MR1174159

[5] J Cheeger, On the spectral geometry of spaces with cone-like singularities, Proc. Nat. Acad. Sci. U.S.A. 76 (1979) 2103-2106 MR530173

[6] J Cheeger, On the Hodge theory of Riemannian pseudomanifolds, from: "Geometry of the Laplace operator (Proc. Sympos. Pure Math., Univ. Hawaii, Honolulu, Hawaii, 1979)", Proc. Sympos. Pure Math., XXXVI, Amer. Math. Soc., Providence, R.I. (1980) 91-146 MR573430

[7] J Cheeger, $\mathbf{X}$ Dai, $L^{2}$-cohomology of a non-isolated conical singularity and nonmultiplicativity of the signature, preprint

[8] S S Chern, F Hirzebruch, J-P Serre, On the index of a fibered manifold, Proc. Amer. Math. Soc. 8 (1957) 587-596 MR0087943

[9] PE Conner, The Neumann's problem for differential forms on Riemannian manifolds, Mem. Amer. Math. Soc. 1956 (1956) 56 MR0078467

[10] X Dai, Adiabatic limits, nonmultiplicativity of signature, and Leray spectral sequence, J. Amer. Math. Soc. 4 (1991) 265-321 MR1088332

[11] M Goresky, R MacPherson, Intersection homology theory, Topology 19 (1980) 135162 MR572580

[12] M Goresky, R MacPherson, Intersection homology. II, Invent. Math. 72 (1983) 77129 MR696691

[13] M Goresky, R MacPherson, Stratified Morse theory, Ergebnisse der Mathematik und ihrer Grenzgebiete (3) 14, Springer, Berlin (1988) MR932724

[14] N Habegger, L Saper, Intersection cohomology of cs-spaces and Zeeman's filtration, Invent. Math. 105 (1991) 247-272 MR1115543

[15] T Hausel, E Hunsicker, R Mazzeo, Hodge cohomology of gravitational instantons, Duke Math. J. 122 (2004) 485-548 MR2057017

[16] E Hunsicker, R Mazzeo, Harmonic forms on manifolds with edges, Int. Math. Res. Not. (2005) 3229-3272 MR2186793

[17] H C King, Topological invariance of intersection homology without sheaves, Topology Appl. 20 (1985) 149-160 MR800845 
[18] R R Mazzeo, R B Melrose, The adiabatic limit, Hodge cohomology and Leray's spectral sequence for a fibration, J. Differential Geom. 31 (1990) 185-213 MR1030670

[19] C McCrory, Zeeman's filtration of homology, Trans. Amer. Math. Soc. 250 (1979) 147-166 MR530047

[20] W Müller, $L^{2}$-index theory, eta invariants and values of $L$-functions, from: "Geometric and topological invariants of elliptic operators (Brunswick, ME, 1988)", Contemp. Math. 105, Amer. Math. Soc., Providence, RI (1990) 145-189 MR1047280

[21] M Nagase, $L^{2}$-cohomology and intersection homology of stratified spaces, Duke Math. J. 50 (1983) 329-368 MR700144

[22] E C Zeeman, Dihomology. III. A generalization of the Poincaré duality for manifolds, Proc. London Math. Soc. (3) 13 (1963) 155-183 MR0153011

[23] S Zucker, $L_{2}$ cohomology of warped products and arithmetic groups, Invent. Math. $70(1982 / 83)$ 169-218 MR684171

Department of Mathematical Sciences, Loughborough University, Leicestershire, LE11 3TU, United Kingdom

maeh@lboro.ac.uk

http://www-staff. Iboro.ac.uk/ maeh/

Proposed: Lothar Goettsche

Seconded: Jim Bryan, Steve Ferry
Received: 17 February 2006

Revised: 20 Nov 2006 